\title{
Gas permeation through single-crystal ZIF-8 membranes
}

Chen Chen ${ }^{1}$, Aydin Ozcan ${ }^{2}$, A. Ozgur Yazaydin ${ }^{2}$, and Bradley P. Ladewig ${ }^{1,3 *}$

${ }^{1}$ Barrer Centre, Department of Chemical Engineering, Imperial College London, SW7 2AZ, United Kingdom

${ }^{2}$ Department of Chemical Engineering, University College London, WC1E 7JE, United Kingdom

${ }^{3}$ Institute for Micro Process Engineering, Karlsruhe Institute of Technology, Hermann-vonHelmholtz-Platz 1, Eggenstein-Leopoldshafen, 76344, Germany *e-mail: b.ladewig@imperial.ac.uk

\begin{abstract}
Grain boundaries are an unavoidable microstructural feature in intergrown polycrystalline metal-organic framework (MOF) membranes. They have been suspected to be less sizeselective than a MOF's micropores, resulting in suboptimal separation performances - a speculation recently confirmed by transmission electron microscopy of MOF ZIF-8. Singlecrystal membranes, without grain boundaries, should confine mass transport to micropores and reflect the intrinsic selectivity of the porous material. Here, we demonstrate the feasibility of fabricating single-crystal MOF membranes and directly measuring gas permeability through such a membrane using ZIF-8 as an exemplary MOF. Our single-crystal ZIF-8 membranes achieved ideal selectivities up to $28.9,10.0,40.1$ and 3.6 for gas pairs $\mathrm{CO}_{2} / \mathrm{N}_{2}$, $\mathrm{CO}_{2} / \mathrm{CH}_{4}, \mathrm{He} / \mathrm{CH}_{4}$ and $\mathrm{CH}_{4} / \mathrm{N}_{2}$ respectively, much higher than or reversely selective to over 20 polycrystalline ZIF-8 membranes, unequivocally proving the non-selectivity of grain boundaries. The permeability trend obtained in single-crystal membranes aligned with a force field that had been validated against multiple empirical adsorption isotherms.
\end{abstract}

Keywords: metal-organic framework, single crystal, ZIF-8, gas permeation, ideal selectivity 


\section{Introduction}

Metal-organic frameworks (MOFs) are commonly defined as potentially porous coordination polymers made of metal nodes and organic ligands extending in two or three dimensions [1]. Reticular syntheses [2] afforded MOFs a wide range of tailorable pore apertures from $3.0 \AA$ in ZIF-11 [3] to $98 \AA$ in IRMOF-74-XI [4], corresponding to diverse molecular sizes from those of small gases (e.g. hydrogen) to natural proteins. Post-synthetic modifications can usually alter the reactivity of a MOF through its linkers [5]. These two developments have made MOFs strong contenders in adsorption- and membrane-based separations [6, 7]. In the domain of membrane-based gas separations, MOFs are typically used as either the selective layer in polycrystalline pure-MOF membranes [8], or the stability- and permeabilityenhancing filler in mixed-matrix membranes (MMMs) [7, 9]; a few MOFs even simultaneously increase permeability and selectivity of MMMs [10,11].

From a design point of view, polycrystalline pure-MOF membranes are theoretically advantageous over MMMs because a pure-MOF membrane's selectivity should be predictable - close to the optimal, molecular-sieving selectivity of the MOF's pore aperture size [7] - as long as the membrane is properly intergrown and crack-free. Many endeavours have been dedicated to fabricating a crack-free polycrystalline MOF membrane over a substrate by optimising a) chemistry of the precursor solutions [12], b) chemistry of the substrate [13, 14], c) membrane growth method or set-up [15-17], or d) a combination of the aforementioned three $[18,19]$. One of such well-engineered polycrystalline $\mathrm{ZIF}-8\left(\mathrm{Zn}(\mathrm{MeIM})_{2}\right.$, MeIM = 2-methylimidazole) membranes used the pore aperture of ZIF-8 (3.4 $\AA$ ) to score an exceptional separation factor of 105 for propene (kinetic diameter: $4.0 \AA$ ) over propane (kinetic diameter: $4.3 \AA$ ) [12].

Despite these remarkable developments, polycrystalline membranes, by their very nature, always contain grain boundaries that have been suspected to be non-size-selective thus undermining separation performances [6, 20-23] (Fig. 1). A recent breakthrough in 
transmission electron microscopy (TEM) imaging by Zhu et al revealed for the first time the grain boundary structure in a MOF [24]. They recreated the self-assembly process of the interface between two ZIF-8 crystals joint via the (110) plane by molecular dynamics simulation, Two of the three interconnected channels at the grain boundary $(\sim 8.2 \AA$ and $\sim 3.8$ $\AA$ in diameter) were larger than the six-membered-ring channels (3.4 $\AA$ in diameter) of ZIF-8; self- and transport-diffusivities of guest molecules were higher in samples with grain boundaries.

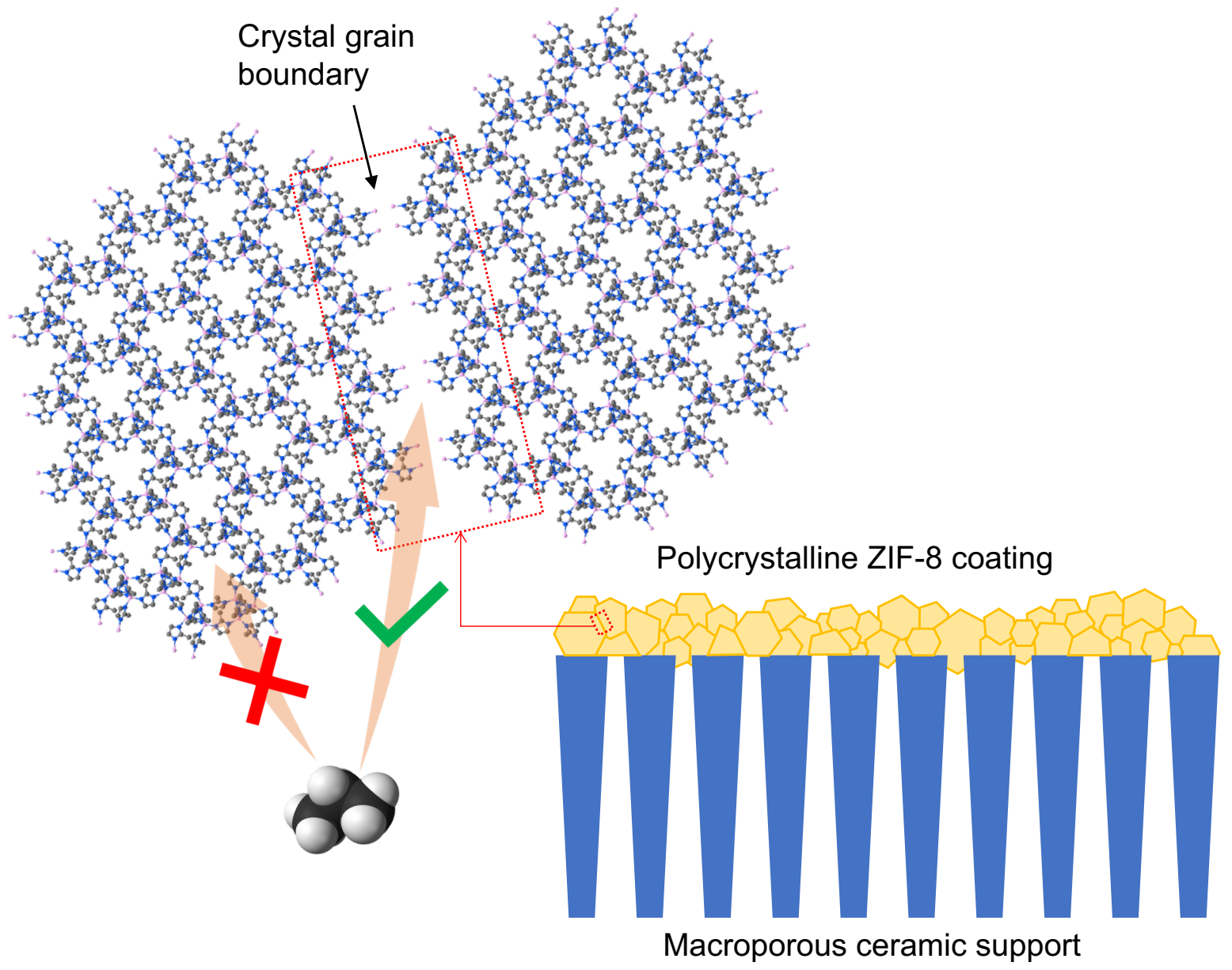

Fig. 1. A schematic diagram showing the possible grain boundary structure in a polycrystalline ZIF-8 membrane. A molecule, such as propane, that is too large to go through the six-membered-ring channel of ZIF-8 may be able to permeate through the larger grain boundary structure (red dotted rectangle). 
Single-crystal membranes (SCMs) are widely recognised as the ideal tool to study a material's intrinsic permeation selectivities [6, 20, 21, 25], and are the assumed model in computational predictions of MOF-membranes' separation performances [26-28]. Single crystals have proved useful for studying intrinsic diffusion properties - infra-red imaging monitored the $\mathrm{CO}_{2}$ uptake and revealed the concentration evolution within a ZIF-8 crystal [29] and at the crystal-polymer interface [30]. However, there are very limited attempts at using single crystals in a membrane configuration to investigate the intrinsic permeation properties because of lack of practical applications of these membranes [31] and fabrication difficulties $[32,33]$. Since single-crystal zeolite membranes fabricated more than three decades ago [32, 34, 35], only one one-dimensional coordination polymer (i.e. by definition, not a MOF [1]) $\left[\mathrm{Cu}_{2}(\mathrm{bza})_{4}(\mathrm{pyz})\right]_{\mathrm{n}}(\mathrm{bza}=\text { benzoate; pyz }=\text { pyrazine })_{[21]}$ and its analogues $[36,37]$ have been made into SCMs. There was, regrettably, no comparison between these SCMs and polycrystalline membranes of the same materials in terms of permeance or selectivity. In our proof-of-concept study of gas permeation through a single-crystal MOF membrane, ZIF-8 was chosen as a representative material because of the readily available data on ceramicsupported polycrystalline ZIF-8 membranes for comparison.

A large crystal is a prerequisite for single-crystal membranes for practical reasons - it would be prohibitively difficult to manually pick up and orient the crystal in the subsequent stage of membrane fabrication. Based on our experience, crystals of at least 100 micrometres are worth being considered for further processing. In order for the downstream pressure to be measured with better accuracy, we needed crystals of a few hundred micrometres - a demanding size as most crystallisation studies aimed to make nanosized crystals [38]; this is also pushing the upper limit of ZIF-8 size reported to date (around $300 \mu \mathrm{m}$ ) - first synthesised by Chmelik et al [39] and reproduced or adapted in many other studies [31, 4045]. Crystal growth is a highly complex and intractable process [46]. It is impossible and beyond the capacity of this work to predict a complete set of parameters that will guarantee 
the unusually large crystals; instead, we built upon methods that have produced the largest ZIF-8 crystals reported.

Here we report permeabilities of light gases (helium, carbon dioxide, nitrogen and methane) through a single-crystal membrane of ZIF-8, and compare the single-crystal ideal selectivities with polycrystalline ZIF-8 membranes. These light gases constitute industrially relevant separations. Carbon dioxide and nitrogen are the main impurities in natural gas [47], which is also an important source of the increasingly demanded helium [26, 48]. Separating $\mathrm{CO}_{2}$ from $\mathrm{N}_{2}$, the largest component of flue gas, is essential to curbing $\mathrm{CO}_{2}$ emission [49]. To the best of our knowledge, this is the first direct measurement of gas permeation through a single-crystal MOF membrane. Without interference from non-selective grain boundaries or inter-crystalline defects, the single-crystal ideal selectivities can be considered intrinsic to ZIF-8 as permeation occurs through micropores only. We believe that intrinsic selectivities are of great interest to the microporous membrane community as these values could provide a benchmark for polycrystalline ZIF-8 membranes' performance.

\section{Experimental}

\subsection{Synthesis of ZIF-8 (Optimised protocol)}

$1.764 \mathrm{~g}(5.93 \mathrm{mmol})$ of zinc nitrate hexahydrate ( $\geq 98 \%$, Sigma-Aldrich) was dissolved in 15 $\mathrm{ml}$ of methanol ( $\geq 99.8 \%$ HiPerSolv CHROMANORM® grade, VWR UK). $0.9739 \mathrm{~g}(11.86$ $\mathrm{mmol})$ of 2-methylimidazole (99\%, Sigma-Aldrich) and $0.4034 \mathrm{~g} \mathrm{(5.93} \mathrm{mmol)} \mathrm{of} \mathrm{sodium}$ formate (99\%, Sigma-Aldrich) were dissolved in $25 \mathrm{ml}$ of methanol. The two solutions were combined and mixed briefly for 5 minutes on a stirring plate. Meanwhile, 3 new 20-ml crimpcap glass vials (Kinesis UK) were rinsed with methanol and set aside with their caps loosely placed over them to prevent dust or any particulates from entering the vials. $13 \mathrm{ml}$ of the combined precursor solution was drawn by a syringe and passed to each glass vial through a 0.2- $\mu \mathrm{m}$ polytetrafluoroethylene (PTFE) syringe filter (VWR UK). The vials were sealed by a manual crimper (Kinesis UK) and placed in a $90-^{\circ} \mathrm{C}$ oven for 24 hours. The vials were left to 
cool naturally in the oven until they were safe to touch. Large crystals on the wall of vials (Fig. S7) were carefully picked up by PTFE tweezers with fine, pointed ends and released into a small snap-cap vial of fresh methanol. Both the manually selected crystals and the leftover crystals were soaked in fresh methanol replaced every 24 hours for 2 days - the extensive solvent exchange was to increase the chance of removing unreacted precursor chemicals. The crystals were dried in a vacuum oven at $25^{\circ} \mathrm{C}$ for 6 hours. The manually selected crystals were inspected under an optical microscope. Single, visually defect- and crack-free crystals with the characteristic dodecahedron shape of ZIF-8 were kept for membrane fabrication and single-crystal XRD. The leftover crystals were kept in another container for powder XRD, nitrogen adsorption and other characterisation studies.

\subsection{PXRD studies}

Powder X-ray diffraction spectra were collected by an PANalytical X'Pert Pro diffractometer with a CuKa X-ray source $(40 \mathrm{kV}, 20 \mathrm{~mA})$ and a reflection-transmission spinner sample stage at ambient conditions. Large crystals were ground before pressed onto the sample holder to obtain a flat surface.

\subsection{Nitrogen sorption}

Nitrogen sorption measurement was performed on a Micromeritics 3Flex volumetric instrument at $77 \mathrm{~K}$. The sample was evacuated at $100{ }^{\circ} \mathrm{C}$ under vacuum overnight, followed by 2 hours of in situ degassing at $100{ }^{\circ} \mathrm{C}$ under vacuum prior to the start of nitrogen sorption analysis. BET surface area was estimated from the quantities adsorbed at relative pressures between 0.045 and 0.299 .

\subsection{Construction of single-crystal membranes}

We adapted the single-crystal zeolite membrane model designed by Geus et al [35] because of its simplicity and compatibility with a commercially available membrane holder (Merck Millipore, XX4404700). Steel plates (47 mm in diameter, $0.37 \mathrm{~mm}$ in thickness) with a 400 - 
$\mu \mathrm{m}$-diameter hole drilled in the centre were made in-house and cleaned with acetone. The construction was carried out under an optical microscope. Epoxy (Araldite, rapid 2component epoxy) was spread around the hole and pushed to the rim of the hole as closely as possible without going over the rim. Five minutes later, a ZIF-8 crystal, held by highprecision plastic tweezers (ideal-tek, 707A.DG), was placed over the epoxy with its large flat base parallel to the steel plate, and secured in place by a gentle push. We allowed the first layer of epoxy to partially set so that it would not overflow the rim of the hole and cover the bottom of the crystal. A fresh batch of epoxy was made and spread around the crystal to ensure a gas-tight seal. The membrane assembly was left at ambient conditions overnight for the epoxy to cure.

\subsection{Gas permeation measurements}

The constant-volume, pressure-rise apparatus (Fig. S5) was used to measure gas permeation. After the membrane was placed in the permeation cell and sealed by a Viton Oring, each segment of the rig was evacuated for 5 to 30 minutes depending on the size of the segment. Gas was introduced to the upstream tank until the pressure stabilised at around 2.1 bar absolute (or 31 psia). The system was left in this state for 2 days, which we observed was enough for the downstream air ingress to reach a steady-state, whilst the pressure gauges 1 and 2 (Fig. S5) were recording continuously. The valve V-2 was then opened; the upstream and downstream pressures were recorded for another 2 days.

\section{Results}

\subsection{Synthesis of large, single crystals of ZIF-8}

The plethora of chemical and process variables used to synthesise the same MOF makes it challenging to systemically and extensively investigate crystallisation processes. For example, ZIF-8 have been made from various $\mathrm{Zn}^{2+}$ salts (nitrate, acetate, sulfate, chloride, bromide, iodide, perchlorate etc) $[12,50,51]$, with or without $[52,53]$ a modulator, in different solvents (water, alcohols, dimethylformamide, dimethyl sulfoxide, acetone etc) [52-54] with 
different molar ratios of reactants at a range of temperatures [55]. To further complicate the matter, the variables may not be independent from each other - one could behave differently when combined with different sets of other variables. For example, sodium formate, a common modulator in ZIF-8 syntheses, was observed to accelerate nucleation and result in smaller ZIF-8 crystals in a solvothermal synthesis [41]; the same modulator served the opposite purpose (i.e. slowing down nucleation and facilitating the formation of bigger ZIF-8 crystals) in an ambient-condition synthesis with stirring [40]. Some studies have tried to control the crystal size of ZIF-8 by adjusting a few variables $[50,56]$; however, the large array of variables means our understanding of crystal size engineering is merely fragments of the whole picture. In view of these challenges, it is more realistic to fine-tune existing protocols than designing the optimal one ab initio.

On the basis of the largest-to-date ZIF-8 [31, 39], we formulated a reproducible synthesis protocol that made large single crystals of ZIF-8 up to $1 \mathrm{~mm}$ (Fig. 2a). Most of the crystals that appeared to be single, crack-free under the microscope were above $500 \mu \mathrm{m}$ (Fig. S2a). The optimal protocol was finalised after varying the zinc salt, synthesis temperature and the modulator concentration (Supplementary Information 1.1); the observations did not always agree with what previous studies suggested, highlighting limitations of the current knowledge of crystal size engineering. For example, we consistently obtained larger crystals from zinc nitrate than from zinc chloride, whilst the opposite was reported in the literature $[12,50]$. Also, we postulated that two opposing roles - deprotonation agent and competitive ligand - of the modulator were present instead of just one (Supplementary Information 1.1). Connecting the fragments of existing insights to form the complete picture of ZIF-8 size control is well beyond the capacity of this work; nonetheless, we found that a few simple steps of removing undesired nucleation sites (filtering the precursor solution, using high-purity solvent and rinsing new glass vials with high-purity solvent) helped achieve bigger crystals than the original methods $[31,39]$ and ensure the reproducibility of our protocol. 
(a)

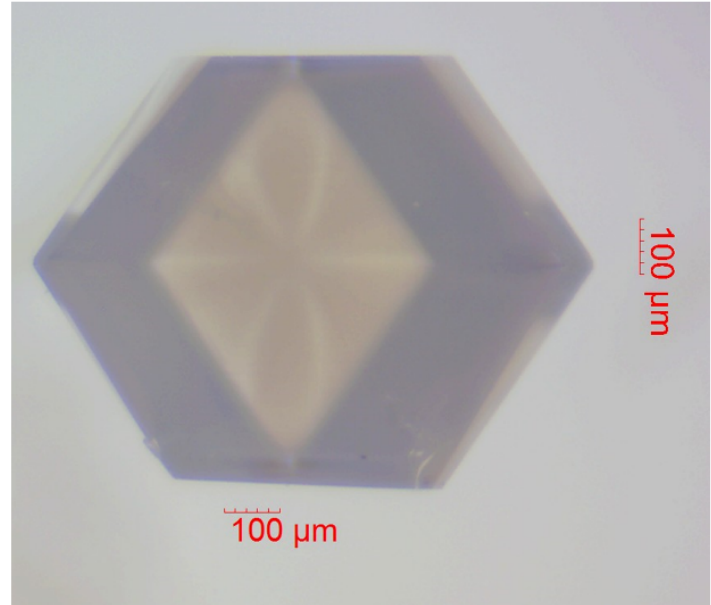

(b)

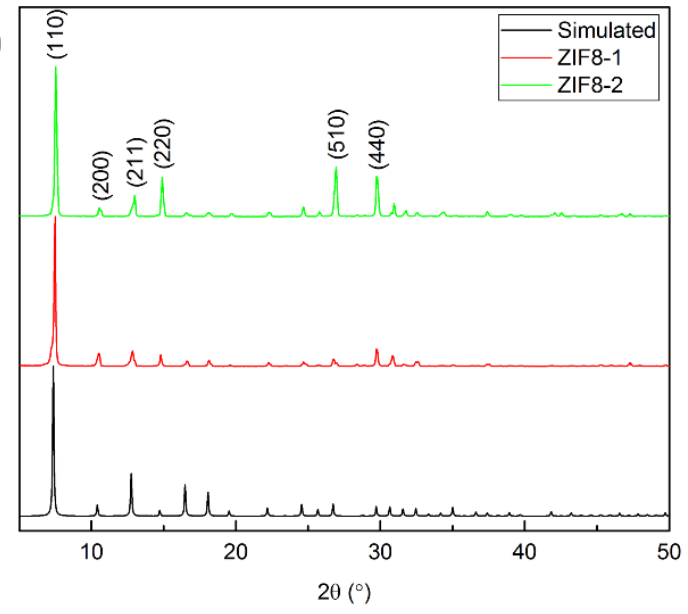

Fig. 2. (a) A micrograph of a millimetre-sized single crystal ZIF-8 viewed along [110] axis. (b) Powder X-ray diffraction (PXRD) pattern of two batches of ZIF-8 from which large, single crystals were selected to make membranes. The two batches were made with the same conditions (in methanol at $90{ }^{\circ} \mathrm{C}$ for 24 hours, i.e. the optimised protocol in Section 2.1). After large, single crystals were isolated, the remaining product was used for PXRD. The simulated pattern was from Park et al [3].

Single-crystal X-ray diffraction (Table S3) and powder X-ray diffraction spectra (Fig. 2b) were collected to confirm that the product was indeed high-quality ZIF-8. A Type 1 adsorption isotherm characteristic of microporous materials [57] was obtained from nitrogen sorption at $77 \mathrm{~K}$ (Fig. S1), from which the Brunauer-Emmett-Teller surface area was calculated to be $1,490 \mathrm{~m}^{2} \mathrm{~g}^{-1}$, within the range of literature values from 960 to $1,918 \mathrm{~m}^{2} \mathrm{~g}^{-1}$ compiled by Huang et al [58].

\subsection{Single-crystal membrane fabrication}

In order to test gas permeation through a SCM in a commercially available permeation test cell (Merck Millipore, XX4404700), we glued the selected single crystal to a steel plate (47 $\mathrm{mm}$ in diameter, $0.37 \mathrm{~mm}$ in thickness) with a $400-\mu \mathrm{m}$-diameter hole drilled in the centre. Two single-crystal ZIF-8 membranes, ZIF8-SCM-1 and ZIF8-SCM-2 (Figs. 3a and 3b) containing single crystals ZIF8-1 and ZIF8-2 respectively, were fabricated and tested. ZIF8-1 
and ZIF8-2 were perfect-looking single crystals of ZIF-8 selected from two identical syntheses detailed in Methods.

Although ZIF-8 is isotropic and has identical six-membered channels along the [111] direction, it has another set of inaccessible four-membered channels in the [100] direction $[18,59]$. Therefore, having the pressure gradient in the [111] direction makes the ideal orientation; and [100] direction, the most unfavourable because of a more torturous permeation path. We did not engineer the growth direction of ZIF-8 crystals. Serendipitously, most of the single crystals harvested from the wall of a glass vial grew along similar directions - somewhat between [111] and [110] - as seen by comparing the Figs. 3a and 3b with Fig. S4. Conveniently, they were 'half crystals' - half of a whole rhombic dodecahedron - with a large, flat surface (Fig. 3b inset) originally in contact with the wall. This large, flat surface became the base parallel to the steel plate and perpendicular to the pressure gradient direction (Fig. 3c). Therefore, harvesting single crystals from the wall of glass vials proved beneficial in two ways - a) it reduced the chance of picking up intergrown crystals, and $b$ ) it helped ensure a rather consistent orientation of the embedded crystal in the membrane assembly. 

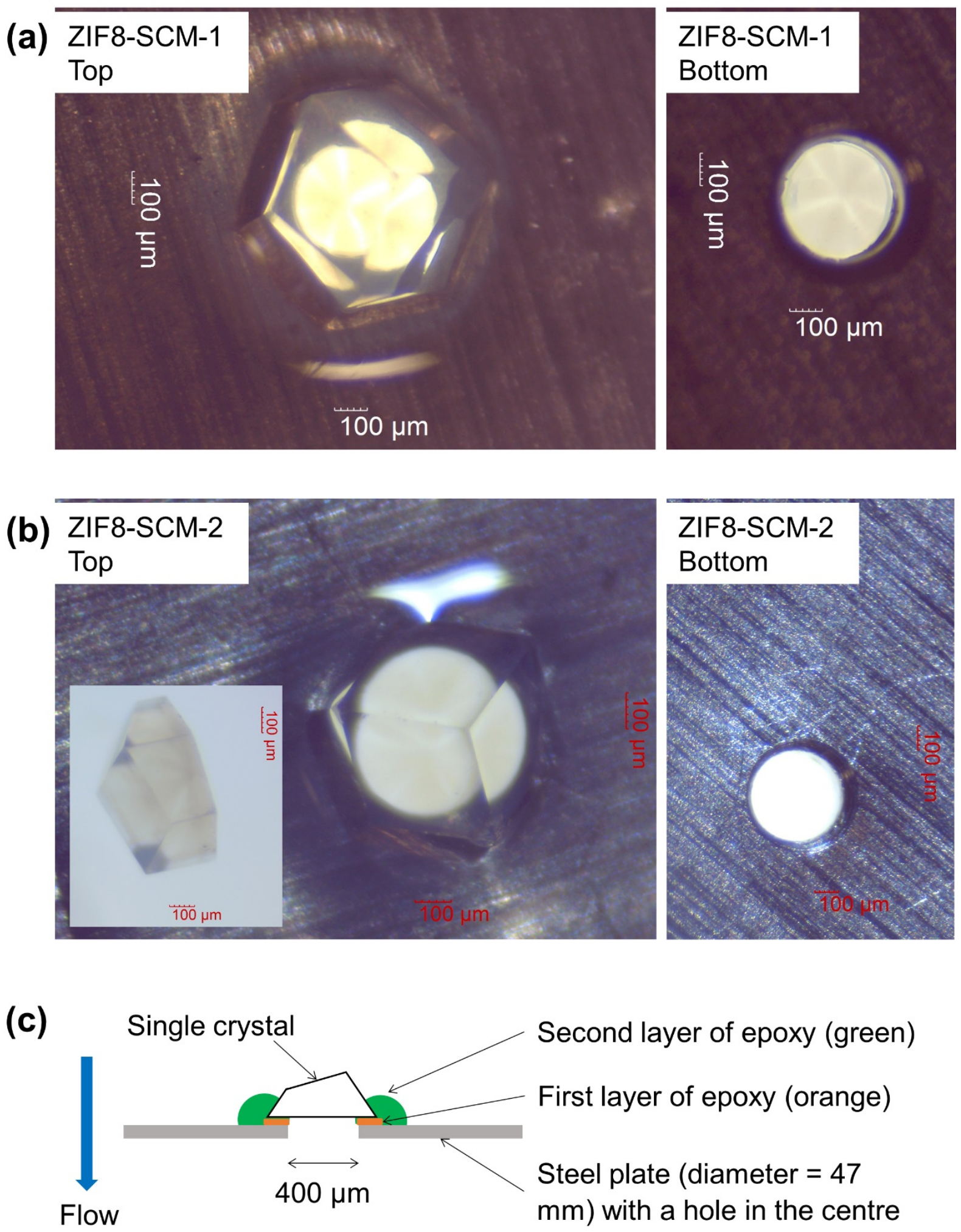

Fig. 3. (a) Micrographs of the top and bottom views of ZIF8-SCM-1. (b) Micrographs of the top and bottom views of ZIF8-SCM-2, inset: the crystal that was embedded in ZIF8-SCM-2. 
(c) A schematic side-view of the single-crystal membrane assembly.

Our SCM assembly has its limitations. Firstly, the embedded crystal does not have a set of parallel surfaces (Fig. 3c), resulting in uneven thickness - the average thickness was used in permeability calculations. There are errors associated with averaging and even the method of using the average thickness (Supplementary Information 1.4.4); however, they will not affect the ideal selectivities of each membrane because when taking the ratio of two permeabilities, the thickness term disappears (Supplementary information 1.4.1). Secondly, the thickness far exceeds what is conventionally acceptable for a membrane. This is the side effect of using a large ZIF-8 crystal as the crystal grows in all three dimensions. We decided not to polish the brittle crystal to protect its integrity. Future work may consider polishing methods that do not damage the crystal, or using MOFs that naturally have a plate-like morphology. Lastly, the extremely small permeation area (i.e. the area of the hole on the steel plate) presents a challenge to the downstream pressure measurement. We kept the downstream volume of the rig (Fig. S5) is kept to the minimum - just the internal volume of the necessary tubing - so that the pressure transducer can pick up any change caused by the small amount of permeate.

\subsection{Single-gas permeation and ideal selectivities}

Single-gas permeabilities of both single-crystal membranes agree with the trend reported by other ZIF-8 membranes - permeability is generally inversely proportional to the kinetic diameter of the permeant, and framework flexibility $[31,60]$ allows transport of molecules larger than the aperture size (Table S1 and Fig. S6). We compared our SCMs' ideal selectivities of four industrially important separations $\left(\mathrm{CO}_{2} / \mathrm{N}_{2}, \mathrm{CO}_{2} / \mathrm{CH}_{4}, \mathrm{He} / \mathrm{CH}_{4}\right.$ and $\left.\mathrm{CH}_{4} / \mathrm{N}_{2}\right)$ with over 20 polycrystalline ZIF-8 membranes that measured single-gas permeation of common small gases at room temperature (Fig. 4 and Table S2). Some polycrystalline ZIF-8 membranes such as the those reported by Brown et al [61] and Marti et al [62] were not included in the comparison because the Torlon polymer substrate showed an ideal $\mathrm{CO}_{2} / \mathrm{N}_{2}$ 
selectivity of 0.90 [63] - higher than the Knudsen value of 0.8 . The macroporous substrate might have affected permeation through adsorption.

Despite the disparity in our two SCMs' ideal selectivities, they are almost always well above the Knudsen selectivities and polycrystalline ZIF-8 membranes' selectivities for all four gas pairs. Most of the polycrystalline selectivities do not reflect a remarkable improvement from some were even below - Knudsen selectivities, suggesting that the molecular sieving potential of ZIF-8 was not fully achieved in those membranes. Interestingly, both SCMs were $\mathrm{CH}_{4}$-selective whereas most polycrystalline ZIF-8 membranes had ideal $\mathrm{CH}_{4} / \mathrm{N}_{2}$ selectivities fluctuating around unity, showing no selectivity or marginal N2-selectivity (Fig. 4d). Two membranes $[64,65]$ showed superior or similar $\mathrm{CO}_{2} / \mathrm{CH}_{4}$ selectivity to ours (Fig. 4b), but neither membrane could match our results in other gas pairs. Thus, their exceptional $\mathrm{CO}_{2} / \mathrm{CH}_{4}$ selectivities should not be generalised to indicate superior membrane performance.

(a)

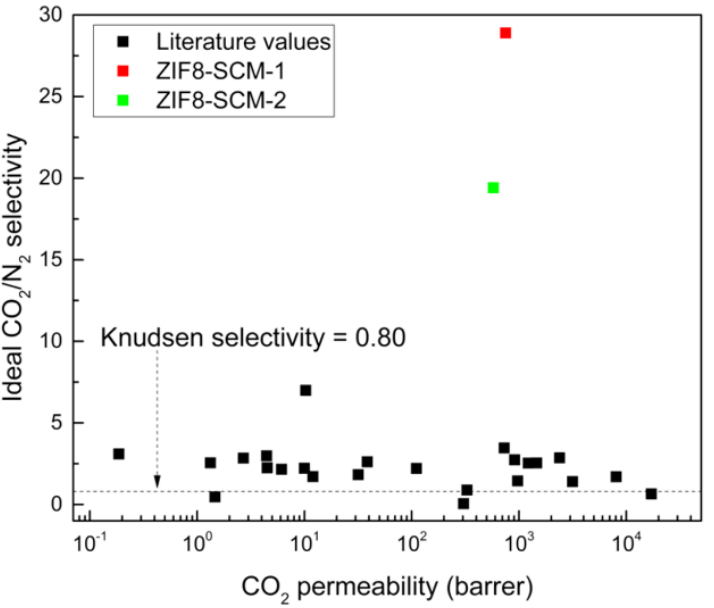

(c)

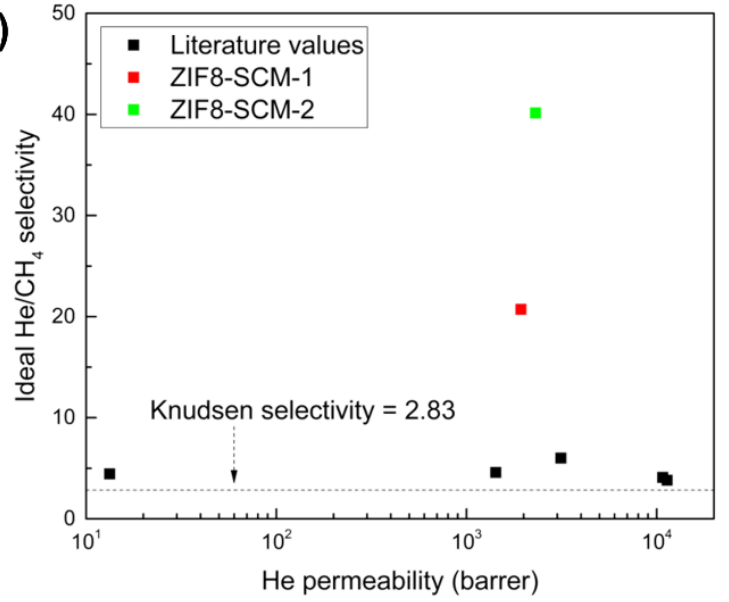

(b)

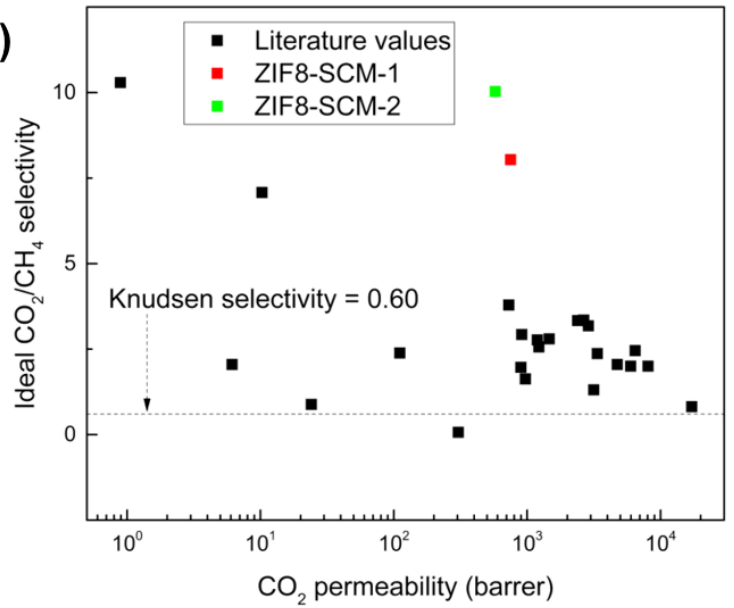

(d)

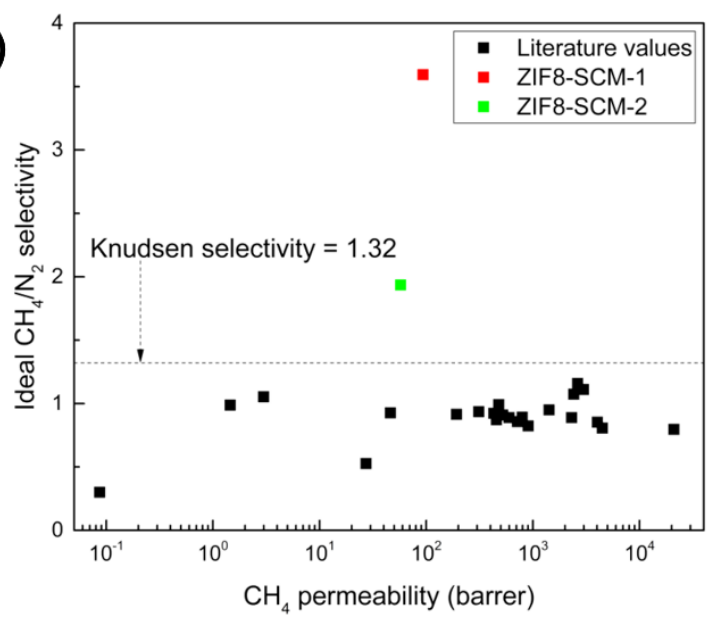


Fig. 4. Single-crystal ZIF-8 membranes' ideal separation performance in (a) $\mathrm{CO}_{2} / \mathrm{N}_{2}$, (b) $\mathrm{CO}_{2} / \mathrm{CH}_{4}$, (c) $\mathrm{He} / \mathrm{CH}_{4}$, and (d) $\mathrm{CH}_{4} / \mathrm{N}_{2}$ at room temperature $\left(20-25^{\circ} \mathrm{C}\right)$. The ideal selectivities are compared with polycrystalline ZIF-8 membranes (Table S2) and Knudsen selectivity. Knudsen selectivity is commonly used to benchmark a membrane's performance - molecular sieving if membrane's selectivity is above the Knudsen selectivity; containing pinholes or mesopores otherwise. There are fewer literature values available for helium as it has not been as extensively tested as other small gases. The effective/average thicknesses of ZIF8-SCM-1 and ZIF8-SCM-2 were around $382 \mu \mathrm{m}$ and $292 \mu \mathrm{m}$ respectively. The method of calculating the average thickness is explained in Supplementary Information 1.4.4 and Fig. S10.

Unlike polycrystalline ZIF-8 membranes that are mostly non-selective or $\mathrm{N}_{2}$-selective over $\mathrm{CH}_{4}$ (Fig. 4d), our SCMs showed reverse selectivity for $\mathrm{CH}_{4}$ over $\mathrm{N}_{2}$. Experimental singlecomponent adsorption isotherms at $25-30{ }^{\circ} \mathrm{C}$ revealed that ZIF-8's uptake of $\mathrm{CH}_{4}$ almost double that of $\mathrm{N}_{2}$ between 0 and 1 bar [66], i.e. the adsorptive $\mathrm{CH}_{4} / \mathrm{N}_{2}$ selectivity is around 2 . The linear isotherms suggest that we can expect the adsorptive selectivity to continue being 2 at our upstream pressure of 2 bar. On the other hand, the small difference in the two molecules kinetic diameters $\left(\mathrm{N}_{2}: 3.64 \AA, \mathrm{CH}_{4}: 3.8 \AA\right)$, coupled with ZIF-8 framework flexibility, means the diffusive selectivity of $\mathrm{N}_{2}$ over $\mathrm{CH}_{4}$ should be marginal. Indeed, the ratio of total diffusion coefficients $\mathrm{N}_{2} / \mathrm{CH}_{4}$ in $\mathrm{ZIF}-8$ was predicted to be 1.38 computationally by Battisti et al [67], giving a diffusive $\mathrm{CH}_{4} / \mathrm{N}_{2}$ selectivity of $1 / 1.38=0.724$. The adsorption-diffusion model suggests that the permeability (or membrane) selectivity is approximately the product of adsorptive and diffusive selectivities $[68,69]$, the permeability selectivity should therefore be approximately $2 \times 0.724=1.45$, making ZIF-8 $\mathrm{CH}_{4}$-selective over $\mathrm{N}_{2}$, in line with our SCMs' performances.

We also compared our ideal selectivities with four sets of in silico results based on four widely used flexible force fields [70-74] for molecular simulations of ZIF-8 (Supplementary 
Information 1.5). Helium was not modelled because of its nearly non-adsorbing nature at room temperature. We believe the force field proposed by Wu et al [71] is more accurate than the other three because it was validated against the experimental adsorption isotherms of $\mathrm{N}_{2}, \mathrm{CO}_{2}$ and $\mathrm{CH}_{4}$ at $298 \mathrm{~K}$ whereas others were only compared with one of the gases. The ideal selectivities obtained from Wu et al's force field (Table S7) agree with our permeability trend of $\mathrm{CO}_{2}>\mathrm{CH}_{4}>\mathrm{N}_{2}$ (Table S1) - an additional validation of their force field. The grain boundary structure may provide an explanation on why polycrystalline ZIF-8 membranes tend to be $\mathrm{N}_{2}$ selective over $\mathrm{CH}_{4}$. There is an extra layer of the 2-methylimidazolate ligands at the grain boundary [24] which are the binding sites for $\mathrm{N}_{2}$ [72]. Therefore, with additional imidazolate rings at grain boundaries, a polycrystalline membrane may have higher $\mathrm{N}_{2}$ adsorption capacity than that in a single crystal, giving a lower and even reverse $\mathrm{CH}_{4} / \mathrm{N}_{2}$ membrane selectivity.

We do not know with absolute certainty what caused the disparities in the two SCMs' selectivities. The disparity is larger when the gas pair involves a slow-permeating gas such as $\mathrm{N}_{2}$ and $\mathrm{CH}_{4}$. A small quantity of ingress air could not be avoided during the extended test period - a method to correct for this is described in the Supplementary Information 1.3. As only a small amount of $\mathrm{N}_{2}$ or $\mathrm{CH}_{4}$ will accumulate in the downstream over the duration of a measurement, it is much more crucial to account for air ingress when testing $\mathrm{N}_{2}$ and $\mathrm{CH}_{4}$ than a fast-permeating species like He. Our method of background subtraction may not be robust enough for $\mathrm{N}_{2}$ and $\mathrm{CH}_{4}$; a high-vacuum set-up in the downstream or a sweep gas in conjunction with a gas chromatography may improve the consistency across different membranes. Having said that, we are aware that it is common to observe nonuniformity among single crystals because of variations in crystal quality [34].

\section{Discussion}

Our work is a proof of concept that direct measurements of gas permeabilities through a single-crystal MOF membrane can be achieved. More importantly, it provided like-for-like 
comparisons between polycrystalline and single-crystal MOF selectivities. The postulation that a single-crystal zeolite membrane may have higher separation factors than the polycrystalline version is not new [32]; however, there was no direct comparison in any SCMs $[21,32,34,35]$ prior to our work. Such comparison was either not part of the objectives of those studies, or unachievable due to the lack of polycrystalline membranes or the lack of data under similar test conditions. By choosing an extensively studied MOF ZIF-8, we showed that by eliminating grain boundaries and constraining gas transport to intracrystalline pores, single-crystal ZIF-8 offered consistently higher ideal selectivities than polycrystalline ZIF-8 membranes for all gas pairs of interest.

This implies that grain boundaries indeed provide a less-selective route than ZIF-8's 6membered-ring channels. With better-engineered synthetic protocols, it is possible to fabricate intergrown, macroscopic-defect-free polycrystalline membranes; but the corollary of a polycrystalline membrane is the presence of grain boundaries. Our results corroborated Zhu et al's conclusion from molecular dynamics simulations of small gases and kinetic vapour adsorption of toluene and 1,3,5-trimethylbenzene in ZIF-8 structures with and without interfacial structures - that the larger interfacial openings in ZIF-8 increase the transport diffusivities of guest molecules. The discovery that larger molecules' mass transport is increased by a greater extent [24] means the diffusive selectivity $D_{j} / D_{j}$ where $D$ is diffusion coefficient, $i$ is the smaller molecule and $j$ the larger, is reduced by grain boundaries. Since permeability selectivity is the product of adsorptive and diffusive selectivities, polycrystalline ZIF-8 membranes will exhibit lower permeability selectivity than a SCM.

\section{Conclusions}

We measured single-gas permeabilities of $\mathrm{He}, \mathrm{CO}_{2}, \mathrm{~N}_{2}$ and $\mathrm{CH}_{4}$ through two single-crystal ZIF-8 membranes and obtained ideal selectivities that are intrinsic to the chemistry and porous structure of ZIF-8 in the absence of other transport routes such as less selective grain boundaries. Our single-crystal membranes displayed consistently higher ideal $\mathrm{CO}_{2} / \mathrm{N}_{2}$, 
$\mathrm{CO}_{2} / \mathrm{CH}_{4}$ and $\mathrm{He} / \mathrm{CH}_{4}$ selectivities of than an overwhelming majority of ceramic-supported polycrystalline membranes reported in the literature, and reverse $\mathrm{CH}_{4} / \mathrm{N}_{2}$ selectivity compared with most polycrystalline membranes. The reverse $\mathrm{CH}_{4} / \mathrm{N}_{2}$ selectivity was supported by simulation and could be explained by the extra imidazole rings, where $\mathrm{N}_{2}$ interacts with strongly, at grain boundaries. It is not our slightest intention to trivialise the breakthroughs in the field of polycrystalline membranes; they are, after all, a beacon of hope for the commercialisation of MOF membranes. What we want to demonstrate through our work is that grain boundaries in ZIF-8 contribute to mass transport and reduce ideal selectivity. With the accuracy of single-crystal membrane measurements improved in the future, they could provide useful empirical results for validation of new force fields.

\section{Acknowledgements}

C.C. thanks Nicholaus Prasetya for assistance with the gas permeation rig. We thank $\mathrm{Dr}$ Andrew White for collecting and refining single-crystal X-ray diffraction data, and staff at the Imperial Chemical Engineering Department Workshop for making the steel plates.

\section{Appendix A. Supporting information}

Supplementary data associated with this article can be found in the online version at: The simulations are available from the repository located at:

\section{https://doi.org/10.5281/zenodo.1435643}




\section{References}

[1] S.R. Batten, N.R. Champness, X.-M. Chen, J. Garcia-Martinez, S. Kitagawa, L. Öhrström, M. O'Keeffe, M. Paik Suh, J. Reedijk, Terminology of metal-organic frameworks and coordination polymers (IUPAC Recommendations 2013), Pure Appl. Chem., 85 (2013).

[2] O.M. Yaghi, M. O'Keeffe, N.W. Ockwig, H.K. Chae, M. Eddaoudi, J. Kim, Reticular synthesis and the design of new materials, Nature, 423 (2003) 705-714.

[3] K.S. Park, Z. Ni, A.P. Côté, J.Y. Choi, R. Huang, F.J. Uribe-Romo, H.K. Chae, M. O'Keeffe, O.M. Yaghi, Exceptional chemical and thermal stability of zeolitic imidazolate frameworks, Proc. Natl. Acad. Sci, 103 (2006) 10186-10191.

[4] H. Deng, S. Grunder, K.E. Cordova, C. Valente, H. Furukawa, M. Hmadeh, F. Gándara, A.C. Whalley, Z. Liu, S. Asahina, H. Kazumori, M. O'Keeffe, O. Terasaki, J.F. Stoddart, O.M. Yaghi, Large-Pore Apertures in a Series of Metal-Organic Frameworks, Science, 336 (2012) 1018-1023.

[5] H. Furukawa, K.E. Cordova, M. O'Keeffe, O.M. Yaghi, The Chemistry and Applications of Metal-Organic Frameworks, Science, 341 (2013).

[6] J.-R. Li, J. Sculley, H.-C. Zhou, Metal-Organic Frameworks for Separations, Chem. Rev., 112 (2012) 869-932.

[7] M.S. Denny, J.C. Moreton, L. Benz, S.M. Cohen, Metal-organic frameworks for membrane-based separations, Nat. Rev. Mater., 1 (2016) 16078.

[8] A. Bétard, R.A. Fischer, Metal-Organic Framework Thin Films: From Fundamentals to Applications, Chem. Rev., 112 (2012) 1055-1083.

[9] J.E. Bachman, Z.P. Smith, T. Li, T. Xu, J.R. Long, Enhanced ethylene separation and plasticization resistance in polymer membranes incorporating metal-organic framework nanocrystals, Nat. Mater., 15 (2016) 845.

[10] G. Liu, V. Chernikova, Y. Liu, K. Zhang, Y. Belmabkhout, O. Shekhah, C. Zhang, S. Yi, M. Eddaoudi, W.J. Koros, Mixed matrix formulations with MOF molecular sieving for key energy-intensive separations, Nat. Mater. , 17 (2018) 283-289.

[11] J. Dechnik, C.J. Sumby, C. Janiak, Enhancing Mixed-Matrix Membrane Performance with Metal-Organic Framework Additives, Cryst. Growth Des., 17 (2017) 4467-4488.

[12] G. Ramu, M. Lee, H.K. Jeong, Effects of zinc salts on the microstructure and performance of zeolitic-imidazolate framework ZIF-8 membranes for propylene/propane separation, Microporous Mesoporous Mater., 259 (2018) 155-162.

[13] M. Navarro, B. Seoane, E. Mateo, R. Lahoz, G.F. de la Fuente, J. Coronas, ZIF-8 micromembranes for gas separation prepared on laser-perforated brass supports, J. Mater. Chem. A, 2 (2014) 11177-11184.

[14] M.C. McCarthy, V. Varela-Guerrero, G.V. Barnett, H.-K. Jeong, Synthesis of Zeolitic Imidazolate Framework Films and Membranes with Controlled Microstructures, Langmuir, 26 (2010) 14636-14641.

[15] O. Shekhah, R. Swaidan, Y. Belmabkhout, M. du Plessis, T. Jacobs, L.J. Barbour, I. Pinnau, M. Eddaoudi, The liquid phase epitaxy approach for the successful construction of ultra-thin and defect-free ZIF-8 membranes: pure and mixed gas transport study, Chem. Commun., 50 (2014) 2089-2092.

[16] Y. Pan, Z. Lai, Sharp separation of C2/C3 hydrocarbon mixtures by zeolitic imidazolate framework-8 (ZIF-8) membranes synthesized in aqueous solutions, Chem. Commun., 47 (2011) 10275-10277.

[17] R. Ameloot, F. Vermoortele, W. Vanhove, M.B.J. Roeffaers, B.F. Sels, D.E. De Vos, Interfacial synthesis of hollow metal-organic framework capsules demonstrating selective permeability, Nat. Chem., 3 (2011) 382.

[18] O. Shekhah, M. Eddaoudi, The liquid phase epitaxy method for the construction of oriented ZIF-8 thin films with controlled growth on functionalized surfaces, Chem. Commun., 49 (2013) 10079-10081.

[19] H.T. Kwon, H.-K. Jeong, A.S. Lee, H.S. An, T. Lee, E. Jang, J.S. Lee, J. Choi, Defectinduced ripening of zeolitic-imidazolate framework ZIF-8 and its implication to vapor-phase membrane synthesis, Chem. Commun., 52 (2016) 11669-11672. 
[20] S. Qiu, M. Xue, G. Zhu, Metal-organic framework membranes: from synthesis to separation application, Chem. Soc. Rev., 43 (2014) 6116-6140.

[21] S. Takamizawa, Y. Takasaki, R. Miyake, Single-Crystal Membrane for Anisotropic and Efficient Gas Permeation, J. Am. Chem. Soc., 132 (2010) 2862-2863.

[22] Y.-S. Li, F.-Y. Liang, H. Bux, A. Feldhoff, W.-S. Yang, J. Caro, Molecular Sieve Membrane: Supported Metal-Organic Framework with High Hydrogen Selectivity, Angew. Chem. Int. Ed., 49 (2010) 548-551.

[23] J. Gascon, F. Kapteijn, Metal-Organic Framework Membranes-High Potential, Bright Future?, Angew. Chem. Int. Ed., 49 (2010) 1530-1532.

[24] Y. Zhu, J. Ciston, B. Zheng, X. Miao, C. Czarnik, Y. Pan, R. Sougrat, Z. Lai, C.E. Hsiung, K. Yao, I. Pinnau, M. Pan, Y. Han, Unravelling surface and interfacial structures of a metalorganic framework by transmission electron microscopy, Nat. Mater., 16 (2017) 532-536.

[25] B. Liu, Metal-organic framework-based devices: separation and sensors, J. Mater. Chem., 22 (2012) 10094-10101.

[26] O. Kadioglu, S. Keskin, Efficient separation of helium from methane using MOF membranes, Sep. Purif. Technol., 191 (2018) 192-199.

[27] G. Yilmaz, A. Ozcan, S. Keskin, Computational screening of ZIFs for CO2 separations, Mol. Simul., 41 (2015) 713-726.

[28] E. Atci, I. Erucar, S. Keskin, Adsorption and Transport of $\mathrm{CH} 4, \mathrm{CO} 2, \mathrm{H} 2$ Mixtures in a Bio-MOF Material from Molecular Simulations, J. Phys. Chem. C, 115 (2011) 6833-6840. [29] C. Chmelik, Characteristic features of molecular transport in MOF ZIF-8 as revealed by IR microimaging, Microporous Mesoporous Mater., 216 (2015) 138-145.

[30] S. Hwang, R. Semino, B. Seoane, M. Zahan, C. Chmelik, R. Valiullin, M. Bertmer, J. Haase, F. Kapteijn, J. Gascon, G. Maurin, J. Karger, Revealing the Transient Concentration of $\mathrm{CO} 2$ in a Mixed-Matrix Membrane by IR Microimaging and Molecular Modeling, Angew. Chem. Int. Ed. Engl., 57 (2018) 5156-5160.

[31] C. Zhang, R.P. Lively, K. Zhang, J.R. Johnson, O. Karvan, W.J. Koros, Unexpected Molecular Sieving Properties of Zeolitic Imidazolate Framework-8, J. Phys. Chem. Lett., 3 (2012) 2130-2134.

[32] J.E. Lewis, G.R. Gavalas, M.E. Davis, Permeation studies on oriented single-crystal ferrierite membranes, AIChE J., 43 (1997) 83-90.

[33] D.B. Shah, H.Y. Liou, Time-lag measurements for diffusion of aromatics through a silicalite membrane, Zeolites, 14 (1994) 541-548.

[34] D.L. Wernick, E.J. Osterhuber, Permeation through a single crystal of Zeolite NaX, J. Membr. Sci., 22 (1985) 137-146.

[35] E.R. Geus, A.E. Jansen, J.C. Jansen, J. Schoonman, H. van Bekkum, Permeability Studies on a Silicalite Single Crystal Membrane Model, Stud. Surf. Sci. Catal., 65 (1991) 457-466.

[36] Y. Takasaki, S. Takamizawa, Gas Permeation in a Molecular Crystal and Space Expansion, J. Am. Chem. Soc., 136 (2014) 6806-6809.

[37] Y. Takasaki, S. Takamizawa, A preferable molecular crystal membrane for $\mathrm{H} 2$ gas separation, Chem. Commun., 50 (2014) 5662-5664.

[38] F. Gandara, T.D. Bennett, Crystallography of metal-organic frameworks, IUCrJ, 1 (2014) 563-570.

[39] C. Chmelik, H. Bux, J. Caro, L. Heinke, F. Hibbe, T. Titze, J. Kärger, Mass Transfer in a Nanoscale Material Enhanced by an Opposing Flux, Phys. Rev. Lett., 104 (2010) 085902.

[40] J. Cravillon, R. Nayuk, S. Springer, A. Feldhoff, K. Huber, M. Wiebcke, Controlling Zeolitic Imidazolate Framework Nano- and Microcrystal Formation: Insight into Crystal Growth by Time-Resolved In Situ Static Light Scattering, Chem. Mater., 23 (2011) 21302141.

[41] J. Cravillon, C.A. Schroder, H. Bux, A. Rothkirch, J. Caro, M. Wiebcke, Formate modulated solvothermal synthesis of ZIF-8 investigated using time-resolved in situ X-ray diffraction and scanning electron microscopy, CrystEngComm, 14 (2012) 492-498. 
[42] A.-K. Pusch, T. Splith, L. Moschkowitz, S. Karmakar, R. Biniwale, M. Sant, G.B. Suffritti, P. Demontis, J. Cravillon, E. Pantatosaki, F. Stallmach, NMR studies of carbon dioxide and methane self-diffusion in ZIF-8 at elevated gas pressures, Adsorption, 18 (2012) 359-366. [43] K. Zhang, R.P. Lively, C. Zhang, W.J. Koros, R.R. Chance, Investigating the Intrinsic Ethanol/Water Separation Capability of ZIF-8: An Adsorption and Diffusion Study, J. Phys. Chem. C, 117 (2013) 7214-7225.

[44] H. Bux, C. Chmelik, J.M. van Baten, R. Krishna, J. Caro, Novel MOF-Membrane for Molecular Sieving Predicted by IR-Diffusion Studies and Molecular Modeling, Adv. Mater., 22 (2010) 4741-4743.

[45] C. Chmelik, J. van Baten, R. Krishna, Hindering effects in diffusion of CO2/CH4 mixtures in ZIF-8 crystals, J. Membr. Sci., 397-398 (2012) 87-91.

[46] M.W. Anderson, J.T. Gebbie-Rayet, A.R. Hill, N. Farida, M.P. Attfield, P. Cubillas, V.A. Blatov, D.M. Proserpio, D. Akporiaye, B. Arstad, J.D. Gale, Predicting crystal growth via a unified kinetic three-dimensional partition model, Nature, 544 (2017) 456-459.

[47] Z. Sumer, S. Keskin, Adsorption- and Membrane-Based CH4/N2 Separation Performances of MOFs, Ind. Eng. Chem. Res., 56 (2017) 8713-8722.

[48] F. Cao, C. Zhang, Y. Xiao, H. Huang, W. Zhang, D. Liu, C. Zhong, Q. Yang, Z. Yang, X. Lu, Helium Recovery by a Cu-BTC Metal-Organic-Framework Membrane, Ind. Eng. Chem. Res., 51 (2012) 11274-11278.

[49] J. Yu, L.-H. Xie, J.-R. Li, Y. Ma, J.M. Seminario, P.B. Balbuena, CO2 Capture and Separations Using MOFs: Computational and Experimental Studies, Chem. Rev., 117 (2017) 9674-9754.

[50] A. Schejn, L. Balan, V. Falk, L. Aranda, G. Medjahdi, R. Schneider, Controlling ZIF-8 nano- and microcrystal formation and reactivity through zinc salt variations, CrystEngComm, 16 (2014) 4493-4500.

[51] L. Sheng, F. Yang, C. Wang, J. Yu, L. Zhang, Y. Pan, Comparison of the hydrothermal stability of ZIF-8 nanocrystals and polycrystalline membranes derived from zinc salt variations, Mater. Lett., 197 (2017) 184-187.

[52] E.L. Bustamante, J.L. Fernández, J.M. Zamaro, Influence of the solvent in the synthesis of zeolitic imidazolate framework-8 (ZIF-8) nanocrystals at room temperature, J. Colloid Interface Sci., 424 (2014) 37-43.

[53] Y. Pan, Y. Liu, G. Zeng, L. Zhao, Z. Lai, Rapid synthesis of zeolitic imidazolate framework-8 (ZIF-8) nanocrystals in an aqueous system, Chem. Commun., 47 (2011) 20712073.

[54] X. Feng, T. Wu, M.A. Carreon, Synthesis of ZIF-67 and ZIF-8 crystals using DMSO (Dimethyl Sulfoxide) as solvent and kinetic transformation studies, J. Cryst. Growth, 455 (2016) 152-156.

[55] S. Watanabe, S. Ohsaki, T. Hanafusa, K. Takada, H. Tanaka, K. Mae, M.T. Miyahara, Synthesis of zeolitic imidazolate framework-8 particles of controlled sizes, shapes, and gate adsorption characteristics using a central collision-type microreactor, Chem. Eng. J., 313 (2017) 724-733.

[56] P.Y. Moh, M. Brenda, M.W. Anderson, M.P. Attfield, Crystallisation of solvothermally synthesised ZIF-8 investigated at the bulk, single crystal and surface level, CrystEngComm, 15 (2013) 9672-9678.

[57] P. Webb, C. Orr, C. Micromeritics Instrument, Analytical methods in fine particle technology, Micromeritics Instrument Corp., Norcross, Ga., 1997.

[58] H. Huang, W. Zhang, D. Liu, B. Liu, G. Chen, C. Zhong, Effect of temperature on gas adsorption and separation in ZIF-8: A combined experimental and molecular simulation study, Chem. Eng. Sci., 66 (2011) 6297-6305.

[59] H. Bux, A. Feldhoff, J. Cravillon, M. Wiebcke, Y.-S. Li, J. Caro, Oriented Zeolitic Imidazolate Framework-8 Membrane with Sharp H2/C3H8 Molecular Sieve Separation, Chem. Mater., 23 (2011) 2262-2269.

[60] D. Fairen-Jimenez, S.A. Moggach, M.T. Wharmby, P.A. Wright, S. Parsons, T. Düren, Opening the Gate: Framework Flexibility in ZIF-8 Explored by Experiments and Simulations, J. Am. Chem. Soc., 133 (2011) 8900-8902. 
[61] A.J. Brown, N.A. Brunelli, K. Eum, F. Rashidi, J.R. Johnson, W.J. Koros, C.W. Jones, S. Nair, Interfacial microfluidic processing of metal-organic framework hollow fiber membranes, Science, 345 (2014) 72-75.

[62] A.M. Marti, W. Wickramanayake, G. Dahe, A. Sekizkardes, T.L. Bank, D.P. Hopkinson, S.R. Venna, Continuous Flow Processing of ZIF-8 Membranes on Polymeric Porous Hollow Fiber Supports for CO2 Capture, ACS Appl. Mater. Interfaces, 9 (2017) 5678-5682. [63] K.-S. Jang, H.-J. Kim, J.R. Johnson, W.-g. Kim, W.J. Koros, C.W. Jones, S. Nair, Modified Mesoporous Silica Gas Separation Membranes on Polymeric Hollow Fibers, Chem. Mater., 23 (2011) 3025-3028.

[64] Y. Liu, Y. Peng, N. Wang, Y. Li, J.H. Pan, W. Yang, J. Caro, Significantly Enhanced Separation using ZIF-8 Membranes by Partial Conversion of Calcined Layered Double Hydroxide Precursors, ChemSusChem, 8 (2015) 3582-3586.

[65] A. Huang, Q. Liu, N. Wang, Y. Zhu, J. Caro, Bicontinuous Zeolitic Imidazolate Framework ZIF-8@GO Membrane with Enhanced Hydrogen Selectivity, J. Am. Chem. Soc., 136 (2014) 14686-14689.

[66] J. McEwen, J.-D. Hayman, A. Ozgur Yazaydin, A comparative study of CO2, CH4 and N2 adsorption in ZIF-8, Zeolite-13X and BPL activated carbon, Chem. Phys., 412 (2013) $72-$ 76.

[67] A. Battisti, S. Taioli, G. Garberoglio, Zeolitic imidazolate frameworks for separation of binary mixtures of $\mathrm{CO} 2, \mathrm{CH} 4, \mathrm{~N} 2$ and $\mathrm{H} 2$ : A computer simulation investigation, Microporous Mesoporous Mater., 143 (2011) 46-53.

[68] J. Caro, Are MOF membranes better in gas separation than those made of zeolites?, Curr. Opin. Chem. Eng, 1 (2011) 77-83.

[69] R. Krishna, Describing the Diffusion of Guest Molecules Inside Porous Structures, J. Phys. Chem. C, 113 (2009) 19756-19781.

[70] P. Krokidas, M. Castier, S. Moncho, E. Brothers, I.G. Economou, Molecular Simulation Studies of the Diffusion of Methane, Ethane, Propane, and Propylene in ZIF-8, J. Phys. Chem. C, 119 (2015) 27028-27037.

[71] X. Wu, J. Huang, W. Cai, M. Jaroniec, Force field for ZIF-8 flexible frameworks: atomistic simulation of adsorption, diffusion of pure gases as $\mathrm{CH} 4, \mathrm{H} 2, \mathrm{CO} 2$ and $\mathrm{N} 2, \mathrm{RSC}$ Advances, 4 (2014) 16503-16511.

[72] L. Zhang, Z. Hu, J. Jiang, Sorption-Induced Structural Transition of Zeolitic Imidazolate Framework-8: A Hybrid Molecular Simulation Study, J. Am. Chem. Soc., 135 (2013) 37223728.

[73] B. Zheng, M. Sant, P. Demontis, G.B. Suffritti, Force Field for Molecular Dynamics Computations in Flexible ZIF-8 Framework, J. Phys. Chem. C, 116 (2012) 933-938.

[74] B. Zheng, M. Sant, P. Demontis, G.B. Suffritti, Correction to "Force Field for Molecular Dynamics Computations in Flexible ZIF-8 Framework", J. Phys. Chem. C, 117 (2013) 2466224662. 


\title{
Supplementary Information
}

\section{Gas permeation through single-crystal ZIF-8 membranes}

\author{
Chen Chen ${ }^{1}$, Aydin Ozcan ${ }^{2}$, A. Ozgur Yazaydin², and Bradley P. Ladewig ${ }^{1,3 *}$ \\ ${ }^{1}$ Barrer Centre, Department of Chemical Engineering, Imperial College London, SW7 \\ 2AZ, United Kingdom \\ ${ }^{2}$ Department of Chemical Engineering, University College London, WC1E 7JE, United \\ Kingdom \\ ${ }^{3}$ Institute for Micro Process Engineering, Karlsruhe Institute of Technology, Hermann-von- \\ Helmholtz-Platz 1, Eggenstein-Leopoldshafen, 76344, Germany
}

*e-mail: b.ladewig@imperial.ac.uk 


\section{Supplementary Text}

1.1. Variations in ZIF-8 synthesis protocol

1.2. Epoxy non-permeability check

1.3. Method to account for downstream air ingress

1.4. Calculation details

1.5. Self-diffusion coefficients of $\mathrm{CH}_{4}, \mathrm{CO}_{2}$ and $\mathrm{N}_{2}$ in ZIF-8 from molecular simulations

\section{Supplementary Figures}

Figure S1: Nitrogen isotherms at $77 \mathrm{~K}$.

Figure S2. Micrographs of ZIF-8 crystals made with various changes in precursors at $90^{\circ} \mathrm{C}$.

Figure S3. A micrograph of ZIF-8 crystals made from zinc nitrate hexahydrate at $70{ }^{\circ} \mathrm{C}$.

Figure S4. ZIF-8 from different perspectives.

Figure S5. A schematic diagram of the permeation rig.

Figure S6. Single-gas permeabilities of ZIF-8 single-crystal membranes.

Figure S7. Single crystals of ZIF-8 grown on the wall of a synthesis vial in their mother liquor.

Figure S8. A schematic side-view of the pure-epoxy membrane.

Figure S9. Downstream pressure evolution due to air ingress.

Figure S10. Method of estimating the average crystal thickness.

\section{Supplementary Tables}

Table S1: Single-gas permeabilities and ideal selectivities.

Table S2: Ceramic-supported polycrystalline ZIF-8 membranes reported in the literature and their single-gas permeances at room temperature.

Table S3: Crystallographic data of ZIF-8 from single-crystal X-ray diffraction.

Table S4. Lennard-Jones parameters for $\mathrm{CH}_{4}, \mathrm{CO}_{2}$ and $\mathrm{N}_{2}$ molecules. 
Table S5. Predicted number of $\mathrm{CH}_{4}, \mathrm{CO}_{2}$ and $\mathrm{N}_{2}$ molecules adsorbed per $2 \times 2 \times 2 \mathrm{ZIF}-8$ system at 2 bars and $298 \mathrm{~K}$ for different ZIF-8 force fields from GCMC simulations.

Table S6. Self-diffusion coefficients of $\mathrm{CH}_{4}, \mathrm{CO}_{2}$ and $\mathrm{N}_{2}$ in $\mathrm{ZIF}-8$ calculated for loadings obtained at 2 bars and $298 \mathrm{~K}$ from MD simulations using different ZIF-8 force fields.

Table S7. Simulated ideal selectivities of $\mathrm{CO}_{2} / \mathrm{N}_{2}, \mathrm{CO}_{2} / \mathrm{CH}_{4}$ and $\mathrm{CH}_{4} / \mathrm{N}_{2}$ mixtures in ZIF-8. 


\section{Supplementary text}

\subsection{Variations in ZIF-8 synthesis protocol}

\subsubsection{Zinc chloride as the zinc source}

We replaced zinc nitrate hexahydrate with the same molar amount of zinc chloride $(\geq 98 \%$, Sigma-Aldrich), that is, $5.93 \mathrm{mmol}$ or $0.808 \mathrm{~g}$ of zinc chloride, whilst keeping everything else the same as described in the Method section of the main manuscript. There was no isolated, large crystal on the wall of glass vials; many smaller crystals were rather evenly distributed all over the wall, with the bulk of the product at the bottom. We could not see particularly large single crystals when examining the product under an optical microscope. As seen on the micrograph (Fig. S2), most of the ZIF-8 crystals made from zinc chloride are in the range of $50-100 \mu \mathrm{m}$. The absence of large crystals and the generally smaller crystal sizes than those made from zinc nitrate hexahydrate did not agree the observations in other methanolbased solvothermal or room-temperature syntheses of ZIF-8 [1, 2] where the authors suggested that nitrate, being a more reactive salt than chloride, led to a faster coordination reaction between zinc ions and ligands (i.e. faster nucleation) and smaller crystals.

\subsubsection{Synthesis various amounts of modulator}

Apart from the optimised protocol where the molar ratios of Zn:MelM:formate:methanol = 1:2:1:167, we attempted syntheses with molar ratios of 1:2:0:167, 1:2:0.5:167 and 1:2:2:167 whilst keeping everything else the same as described in the Method section of the main manuscript. Without sodium formate, the precursor solution remained clear and no product was formed even after a week. The same was observed in an unmodulated synthesis using zinc chloride as the zinc source.

When using the molar ratio of Zn:MeIM:formate:methanol = 1:2:0.5:167, the precursor solution remained clear after 24 hours; the final product was harvested at the 43 rd hour mark. Most of the crystals were too small for our microscope to resolve; a few larger crystals 
between 100 and $200 \mu \mathrm{m}$ were also observed (Fig. S2c). On the other hand, with more formate than in the optimised protocol, product was formed after 24 hours (Fig. S2d) but appeared much smaller than what was achieved by the optimised protocol (Fig. S2a).

The observation that using no formate and 0.5-part formate resulted in no crystallisation and a longer lag phase than using 1- or 2-part formate, respectively, suggests that the modulator acts as a base that deprotonates the ligand, accelerating nucleation and growth rates and leading to small crystals [3]. However, the fact that the crystal size went through a maximum at 1-part formate when the amount of formate was varied from 0 to 2 parts made us doubt the role of formate was just to increase nucleation and growth rates. It is possible that the other role of formate - a competitive ligand [3, 4] - was in force simultaneously and generating the opposing effect, i.e. slowing down nucleation and growth and facilitating the formation of larger crystals. We postulate under our synthetic conditions, the modulator formate acts as both a deprotonation agent and a competitive ligand; the crystal size can be maximised when a fine balance is achieved between the two opposing roles. Direct experimental evidence is needed to validate or disprove our postulation.

\subsubsection{Synthesis at other temperatures}

The protocol described in the Method section of the main manuscript was also carried out at $70{ }^{\circ} \mathrm{C}$ and $110^{\circ} \mathrm{C}$. ZIF-8 made at $70{ }^{\circ} \mathrm{C}$ are small and highly intergrown (Fig. S3). Multiple attempts at $110{ }^{\circ} \mathrm{C}$ were unsuccessful because the vapour pressure of methanol at $110{ }^{\circ} \mathrm{C}$ compromised the seal of the crimp caps, and methanol evaporated completely. 


\subsection{Epoxy non-permeability check}

A membrane without a ZIF-8 crystal was constructed (Fig. S8) to check the non-permeability of the epoxy. Freshly mixed epoxy was allowed to partially cure for 3 minutes before spread over the hole of a steel plate. The semi-hardened epoxy was still able to bond to the steel plate, yet viscous enough not to seep through the hole to the other side. Helium, the fastest permeating gas of the four, had a permeability of 26 barrer through the pure epoxy membrane at $24{ }^{\circ} \mathrm{C}$, merely $1.3 \%$ and $1.1 \%$ of the helium permeabilities through ZIF8-SCM1 and ZIF8-SCM-2 respectively (Table S1). It was therefore established that the epoxy resin did not provide an alternative transport route for permeants at our experimental conditions. 


\subsection{Method to account for downstream air ingress}

We observed a continuous rise in the downstream pressure after the downstream and the segment between membrane and Valve V-2 (Fig. S5) were evacuated. Since no leaks were picked up by a helium leak detector, we postulated that the pressure rise was air ingress through fittings that were not rated to attain vacuum sealing. The small downstream volume of $1.2 \mathrm{~cm}^{3}$ made the pressure gauge sensitive to small amounts of gases.

The rate of ingress to the downstream became approximately constant after 1.5 days and remained so at the end of the 4 th day, as indicated by the linear pressure-time curve (Fig. S9). We therefore assumed that by keeping the duration of each permeation under 4 days (2 days of stabilising downstream ingress +2 days of gas permeation), the downstream ingress rate could be regarded constant. The actual rate of pressure rise caused by gas permeation $(d P / d t)_{\text {actual }}$ was calculated by:

$\left(\frac{d P}{d t}\right)_{\text {actual }}=\left(\frac{d P}{d t}\right)_{\text {observed }}-\left(\frac{d P}{d t}\right)_{\text {ingress }}$

where

$(d P / d t)_{\text {actual }}=$ the rate of pressure rise caused by permeant accumulated in the downstream $(d P / d t)_{\text {observed }}=$ the apparent gradient from the pressure-time curved collected during gas permeation, which is the result of both gas permeation and air ingress to the downstream 


\subsection{Calculation details}

\subsubsection{Permeability}

Gas permeability was calculated by the following equation [5]:

$p=\frac{V L}{A} \frac{T_{0}}{(\Delta P)_{\text {avg }} T P_{0}}\left(\frac{d P}{d t}\right)_{\text {actual }}$

where $p$ is the permeability in barrer ( 1 barrer $\left.=10^{-10} \mathrm{~cm}^{3}(\mathrm{STP}) \mathrm{cm} \mathrm{cm}^{-2} \mathrm{~s}^{-1} \mathrm{cmHg}^{-1}\right), V$ is the downstream volume in $\mathrm{cm}^{3}, L$ is the membrane thickness in $\mathrm{cm}, A$ is the permeation area in $\mathrm{cm}^{2}$ which, in our case, is the area of the hole in the centre of a steel plate, $(\Delta P)_{\text {avg }}$ is the average trans-membrane pressure difference in $\mathrm{cmHg}, T$ is operating temperature in $\mathrm{K}$, $(d P / d t)_{\text {actual }}$ is the rate of downstream pressure rise in $\mathrm{cmHg} \mathrm{s}^{-1}$ after accounting for the air ingress rate. $T_{0}$ and $P_{0}$ are the standard state temperature $(273 \mathrm{~K})$ and pressure $(76 \mathrm{cmHg})$ respectively. In our experiments, the downstream pressure was two-orders-of-magnitude smaller than the upstream, therefore we assumed the trans-membrane pressure difference to be constant $\left((\Delta P)_{\text {avg }}\right)$ for the duration of each permeation test.

\subsubsection{Ideal selectivity}

The ideal selectivity of gas $i$ over gas $j\left(\alpha_{i j}\right)$ is the ratio of their permeatilities:

$\alpha_{i / j}=\frac{p_{i}}{p_{j}}=\frac{(\Delta P)_{a v g, j}}{(\Delta P)_{a v g, i}} \times \frac{T_{j}}{T_{i}} \times \frac{\left(\frac{d P}{d t}\right)_{a c t u a l, i}}{\left(\frac{d P}{d t}\right)_{a c t u a l, j}}$

When taking the ratio of two permeabilities, downstream volume, permeation area and membrane thickness disappear. The uncertainties associated with each quantity do not affect the accuracy of the ideal selectivity. Therefore, despite not having the most accurate downstream volume or membrane thickness, we are reasonably confident about the accuracy of our SCMs' ideal selectivities.

\subsubsection{Knudsen selectivity}


Knudsen selectivity, calculated from the ratio of Knudsen diffusion fluxes[6], is:

$\left(\alpha_{i / j}\right)_{\text {Knudsen }}=\sqrt{\frac{M_{j}}{M_{i}}}$

where $M_{i}$ and $M_{j}$ are the formula masses of $i$ and $j$ respectively.

\subsubsection{Membrane thickness}

Since the embedded ZIF-8 crystal does not have a set of parallel surfaces along the direction of pressure gradient, we estimated the average thickness along this direction by using a microscope image processing software (Motic) to trace the boundary of the sideview of the crystal as if it was embedded in the membrane assembly (Fig. S10). After the correct calibration and magnification settings were given to the software, it automatically calculated the cross-sectional area. To measure the length as seen on the crystal side-view that corresponded to the large, flat base glued to the steel plate, we drew a parallel line of the same length. The average crystal thickness in the direction of pressure gradient was estimated by cross-sectional area/length of the base.

\subsubsection{Downstream volume}

The downstream volume is the sum of the internal volumes of downstream fittings. The internal volumes were calculated from the computer-aided design drawings available on the Swagelok website. 


\subsection{Self-diffusion Coefficients of $\mathrm{CH}_{4}, \mathrm{CO}_{2}$ and $\mathrm{N}_{2}$ in $\mathrm{ZIF}-8$}

\section{from Molecular Simulations}

Self-diffusivities of $\mathrm{CH}_{4}, \mathrm{CO}_{2}$ and $\mathrm{N}_{2}$ at $298 \mathrm{~K}$ in ZIF-8 were calculated by running equilibrium molecular dynamics (MD) simulations in the NVT ensemble using the DL_POLY CLASSIC software. Prior to running the MD simulations the numbers of molecules of $\mathrm{CH}_{4}, \mathrm{CO}_{2}$ and $\mathrm{N}_{2}$ adsorbed in ZIF-8 at $298 \mathrm{~K}$ and 2 bars, which is the experimental feed pressure, were predicted by performing grand canonical Monte Carlo (GCMC) simulations using the RASPA molecular simulation package. The predicted loadings were then used in the MD simulations. For both GCMC and MD simulations, a simulation box consisting of ZIF-8 unit cell replicated by $2 \times 2 \times 2$ in the $x, y$ and $z$ directions, respectively, was used. The short-range interactions between the atoms in the system were calculated using the Lennard-Jones (LJ) potential and the long-range electrostatic interactions were computed using the Ewald sum method. Cut-off distance for the LJ interactions and the real part of Ewald sum was set to $13 \AA$. Transferable potentials for phase equilibria (TraPPE) force field was used for modelling the $\mathrm{CH}_{4}, \mathrm{~N}_{2}$ and $\mathrm{CO}_{2}$ gas molecules (Table S4) [7, 8]. To model the ZIF-8 structure we considered flexible force fields developed by Krokidas et al. [9], Wu et al. [10], Zhang et al. [11], and Zheng et al. [12, 13], all of which have been widely used in the literature in molecular simulation studies of ZIF-8.

During the GCMC simulations, the ZIF-8 atoms were held fixed at their crystallographically determined positions. Translation, rotation and insertion/deletion of the gas molecules were sampled with equal probability. Each GCMC simulation was equilibrated for $10^{5}$ cycles followed by a $10^{5}$-cycle production run. A cycle is $N$ steps where $N$ is either 20 or the number of molecules in the system, whichever is greater. The predicted average numbers of molecules adsorbed in ZIF-8 for each force field type are given in Table S5. These were rounded off to the nearest integer and then used in the MD simulations. 
During the MD simulations, ZIF-8 framework was treated fully flexible. A Nose-Hoover thermostat was used to keep the temperature at $298 \mathrm{~K}$. The time step was set to $1 \mathrm{fs}$ and each simulation was equilibrated for $5 \mathrm{~ns}$ and then run for another $40 \mathrm{~ns}$. Trajectories were saved every 1000 steps. Self-diffusivities of $\mathrm{CH}_{4}, \mathrm{CO}_{2}$ and $\mathrm{N}_{2}$ were then calculated by mean squared displacement method using the Einstein relation:

$D_{s}=\lim _{t \rightarrow \infty} \frac{1}{6 t}\left\langle\frac{1}{N} \sum_{i}^{N}\left(r_{i}(t)-r_{i}\left(t_{0}\right)\right)^{2}\right\rangle$

where $N$ is the number of gas molecules in the system, $r_{i}(t)$ is the instantaneous position of the ith gas molecule and $r_{i}\left(t_{0}\right)$ is the initial position of the same molecule. To improve the statistics, multiple origins were used and self-diffusion coefficients were calculated over 30 ns trajectories which are reported for each ZIF-8 force field type in Table S6. 


\section{Supplementary figures}

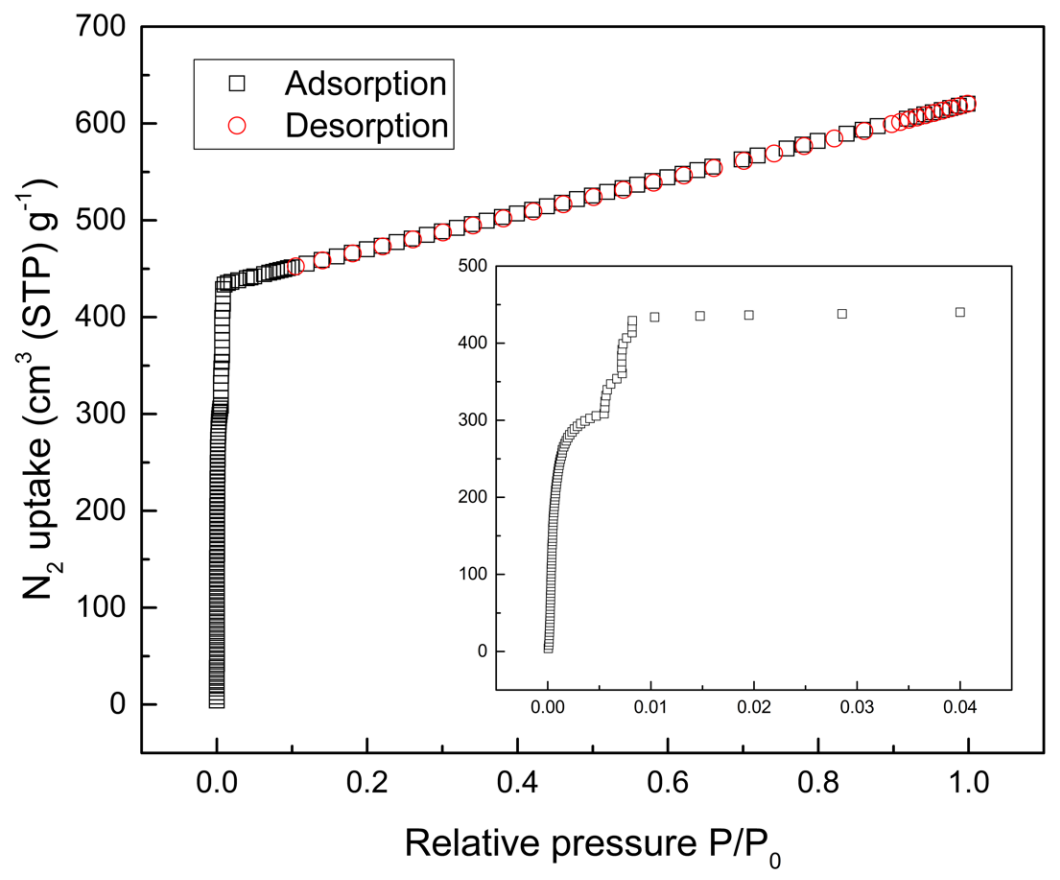

Fig. S1. Nitrogen isotherms at $77 \mathrm{~K}$. The Type 1 adsorption isotherm confirmed the microporous nature of ZIF-8. The inset reveals several adsorption sub-steps at low relative pressures (i.e. micropore region) that were also observed in previous studies [14-16]. Possible reasons for the sub-steps include rearrangement of adsorbed nitrogen molecules [15] and structural changes to ZIF-8 caused by adsorbed nitrogen molecules [16]. 
(a)

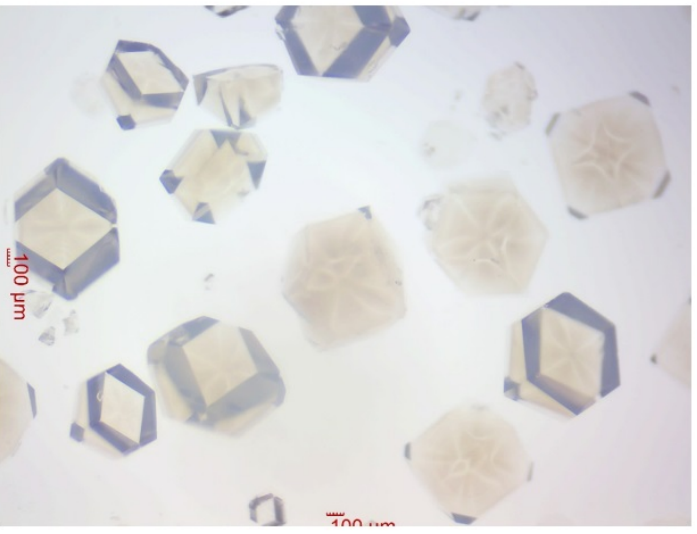

(c)

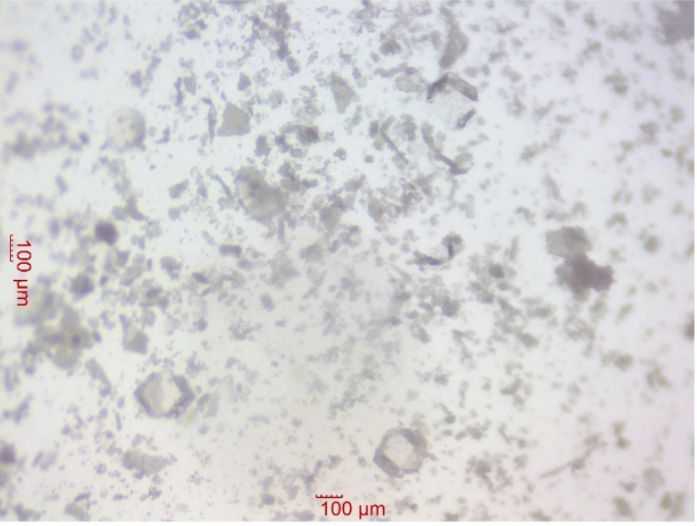

(b)

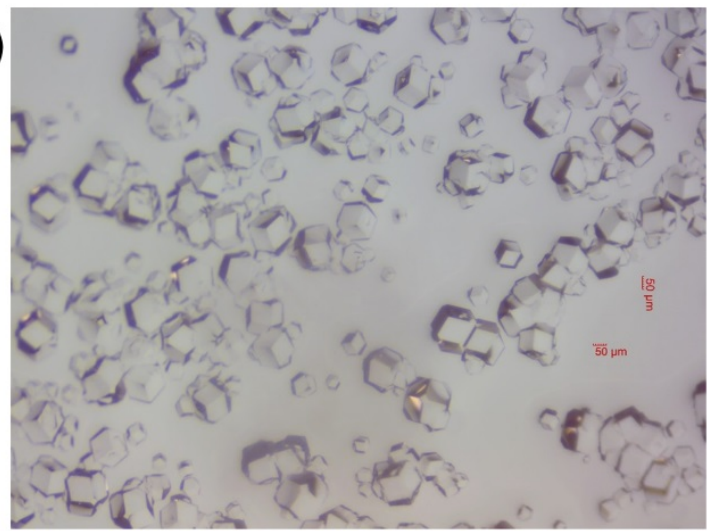

(d)

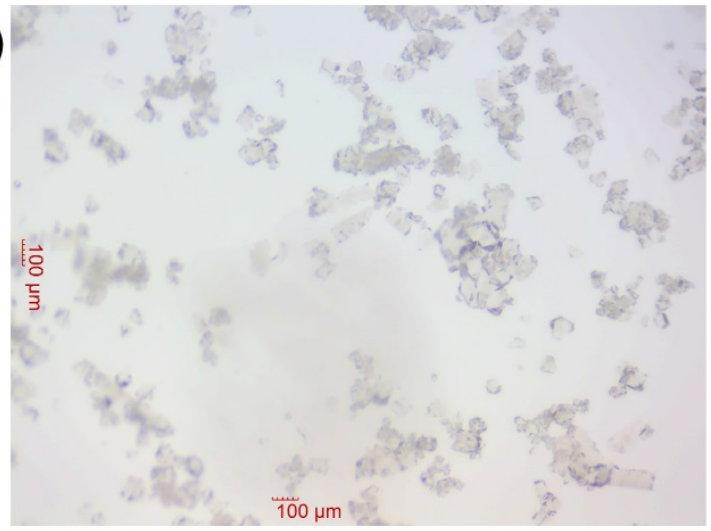

Fig. S2. Micrographs of ZIF-8 crystals made with various changes in precursors at $90^{\circ} \mathrm{C}$. (a) Crystals made by the optimised protocol appear to be high-quality, single crystals above 500 $\mu \mathrm{m}$. (b) Crystals made from zinc chloride are mostly in the range of $50-150 \mu \mathrm{m}$, smaller than those made from zinc nitrate hexahydrate in the optimised protocol. (c) Crystals made using molar ratio Zn:MelM:formate:methanol = 1:2:0.5:167 (i.e. the amount of sodium formate is half of the optimised protocol's) are too small to be examined by our microscope except a few crystals that are between 100 and $200 \mu \mathrm{m}$. (d) Crystals made using molar ratio Zn:MelM:formate:methanol = 1:2:2:167 (i.e. the amount of sodium formate is twice of the optimised protocol's) are small and intergrown. 


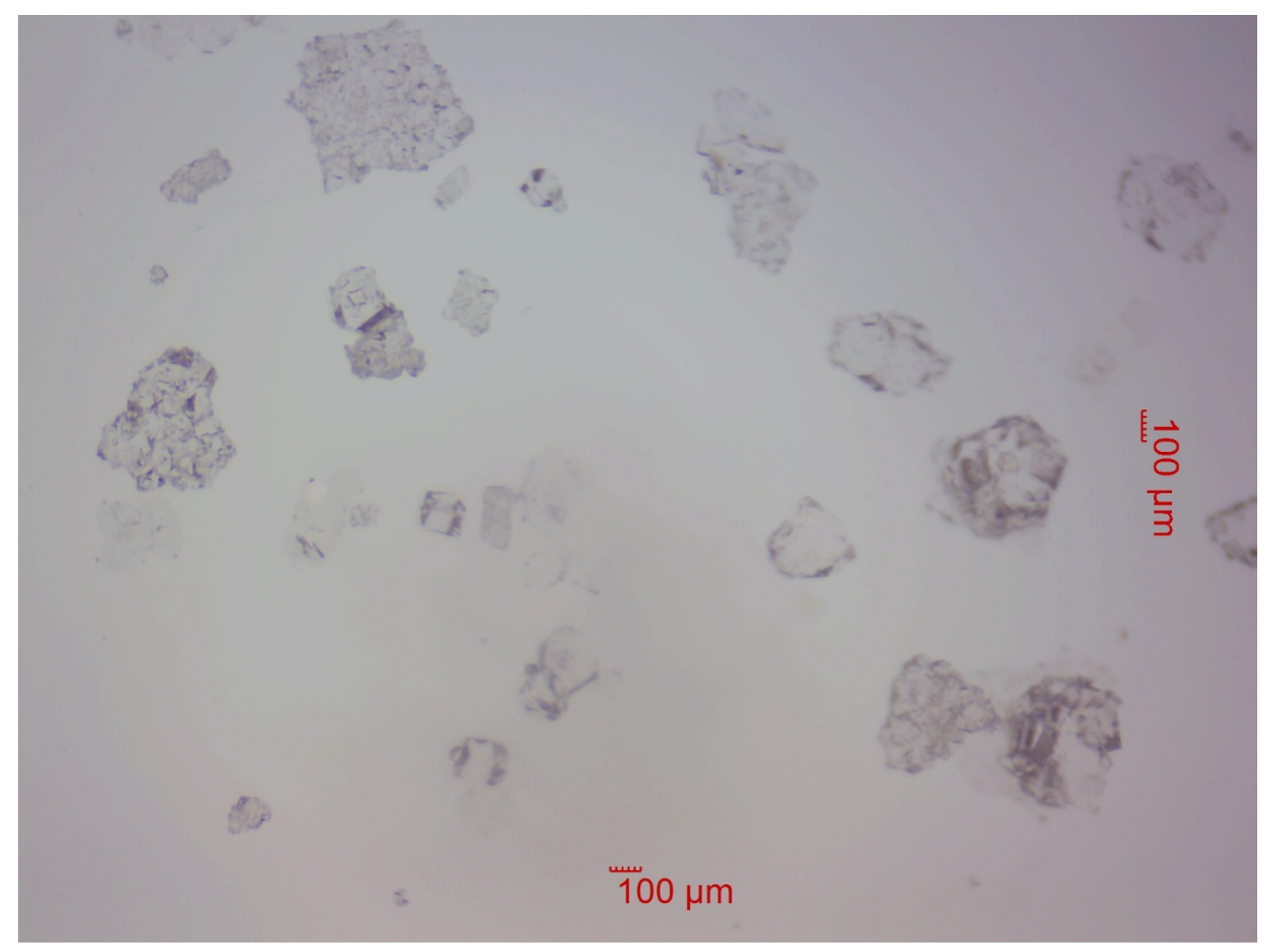

Fig. S3. A micrograph of ZIF-8 crystals made from zinc nitrate hexahydrate at $70{ }^{\circ} \mathrm{C}$. The crystals are small, highly intergrown, or have no well-defined morphology. 
(a)

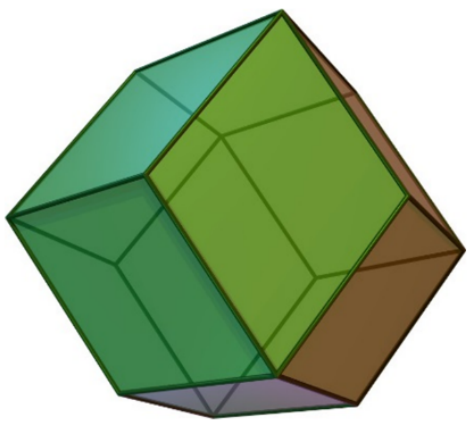

(b)
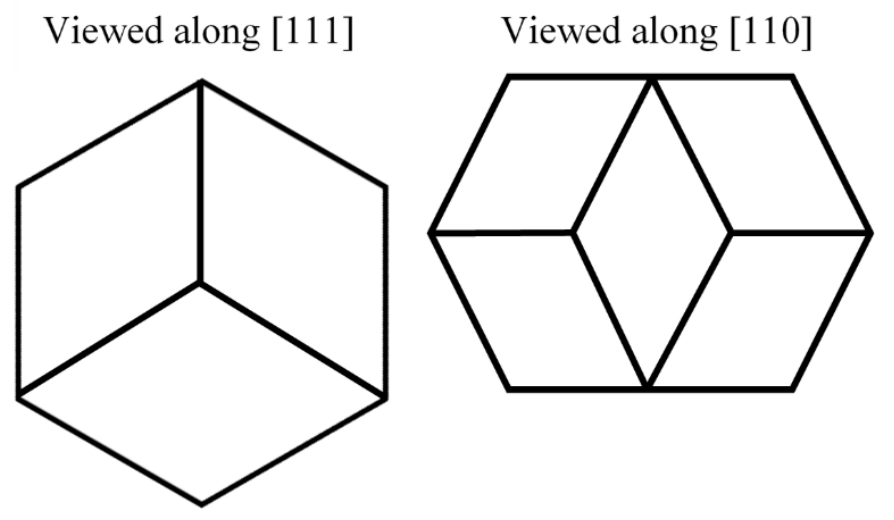

Viewed along [100]

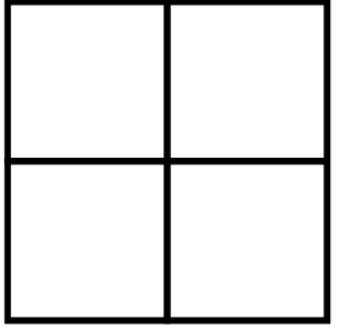

(c)

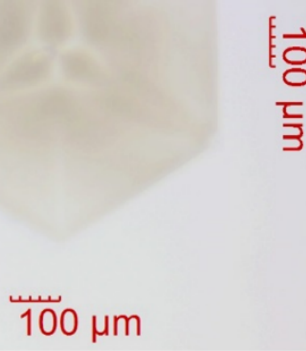

Fig. S4. ZIF-8 from different perspectives. (a) A rhombic dodecahedron, the morphology of

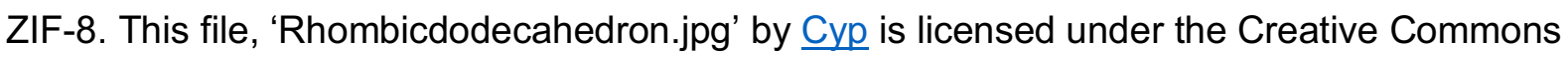
Attribution-Share Alike 3.0 Unported (CC BY-SA 3.0) license. (b) An illustration of the 2dimensional top view when a ZIF-8 crystal is viewed along [111], [110] and [100] directions. (c) Top views of three ZIF-8 'half crystals' grown on the wall of a glass vial with different growth directions. When they lie on their large, flat surface, the top views correspond to the shapes of a rhombic dodecahedron viewed in directions (shown in b) equal to their respective growth directions. 


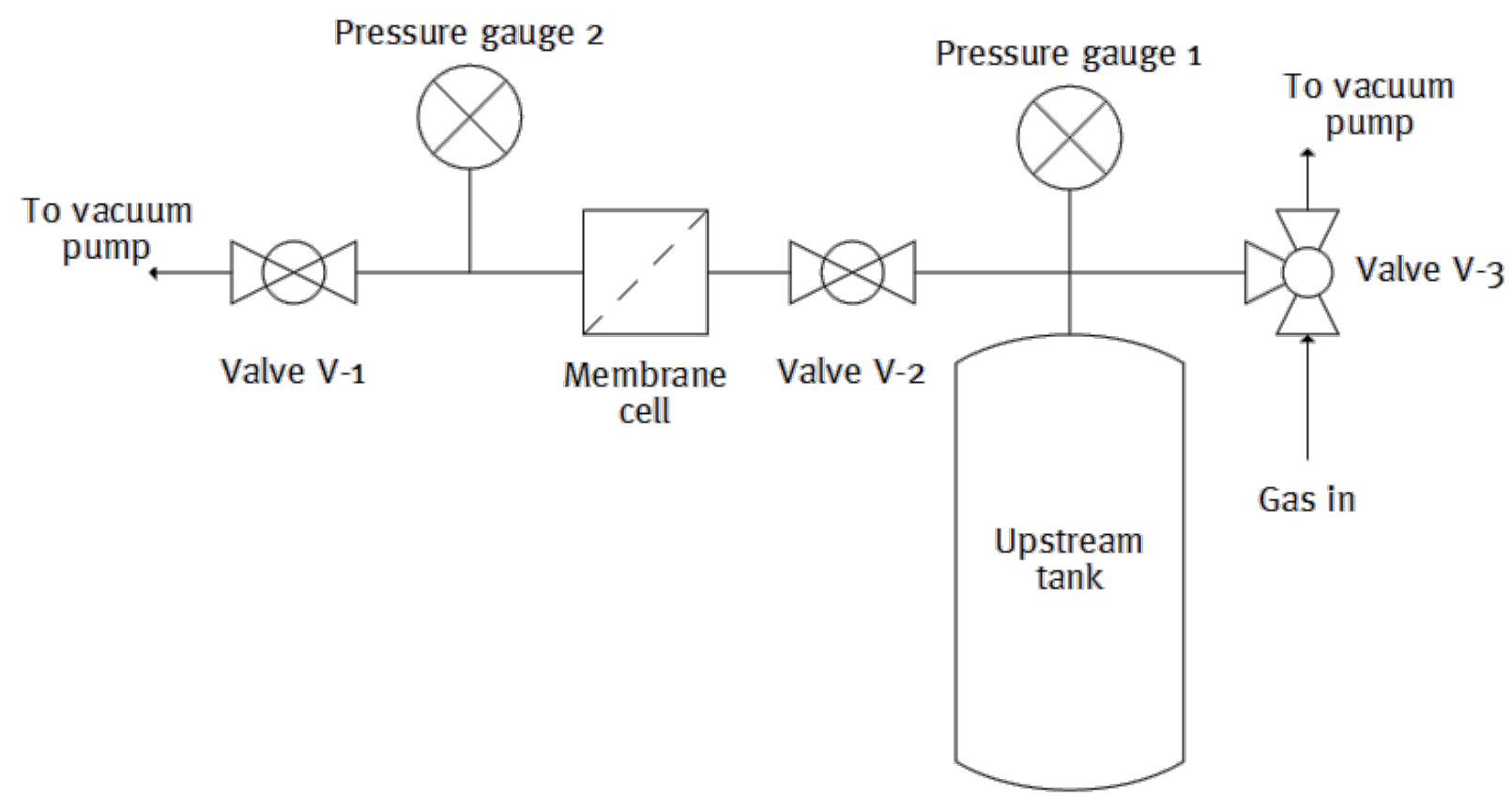

Fig. S5. A schematic diagram of the permeation rig. The downstream of the rig has a constant volume and variable pressure as permeant accumulates in the downstream. 


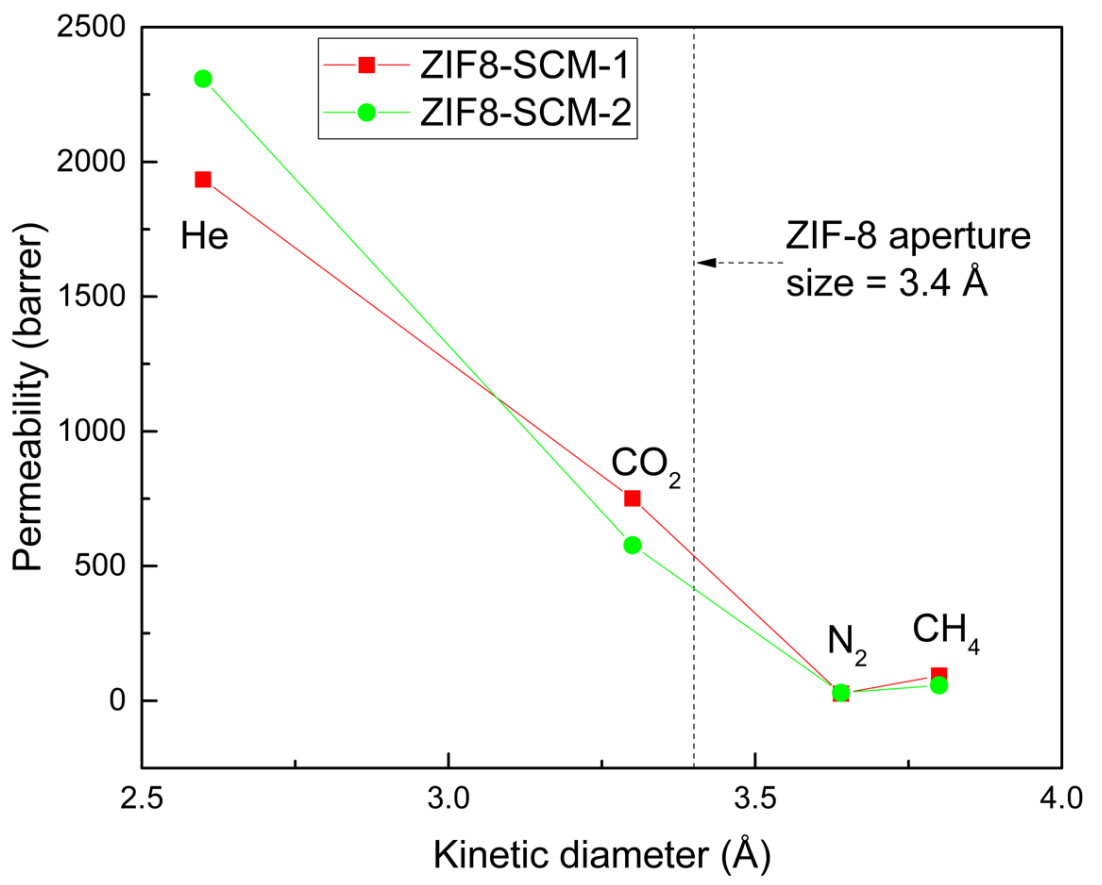

Fig. S6. Single-gas permeabilities of ZIF-8 single-crystal membranes. Experiments were conducted at room temperatures between 20 and $25^{\circ} \mathrm{C} .1$ barrer $=10^{-10} \mathrm{~cm}^{3}(\mathrm{STP}) \mathrm{cm} \mathrm{cm}^{-2}$ $\mathrm{s}^{-1} \mathrm{cmHg}^{-1}$. 


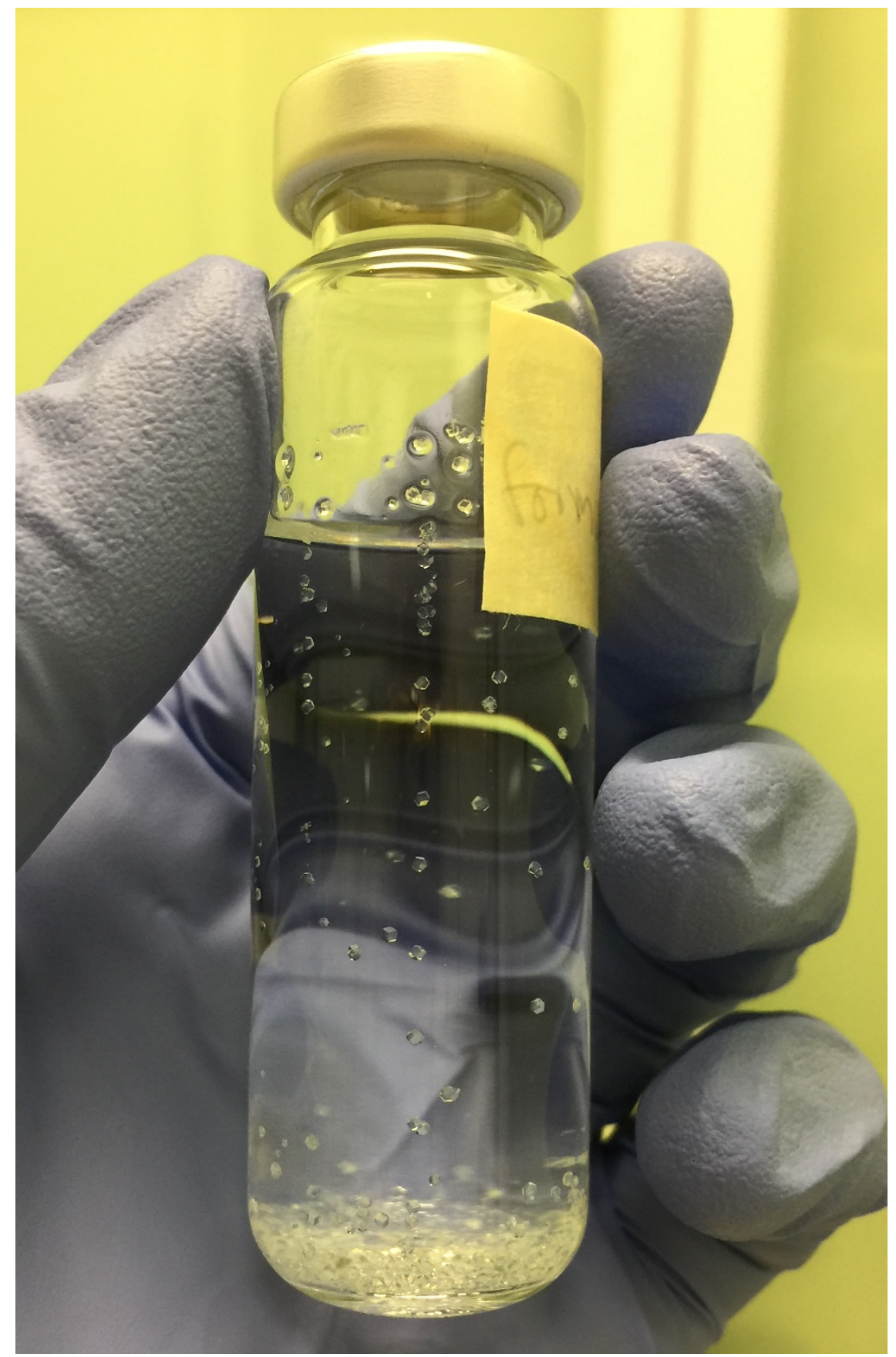

Fig. S7. Single crystals of ZIF-8 grown on the wall of a synthesis vial in their mother liquor. Using the optimised protocol, we had many high-quality, large, single crystals on the wall. Most of the product was inter-grown crystals aggregating at the bottom of the vial; these products were used for PXRD and $\mathrm{N}_{2}$ sorption. For size comparison, the outer diameter of the vial is $23 \mathrm{~mm}$. 


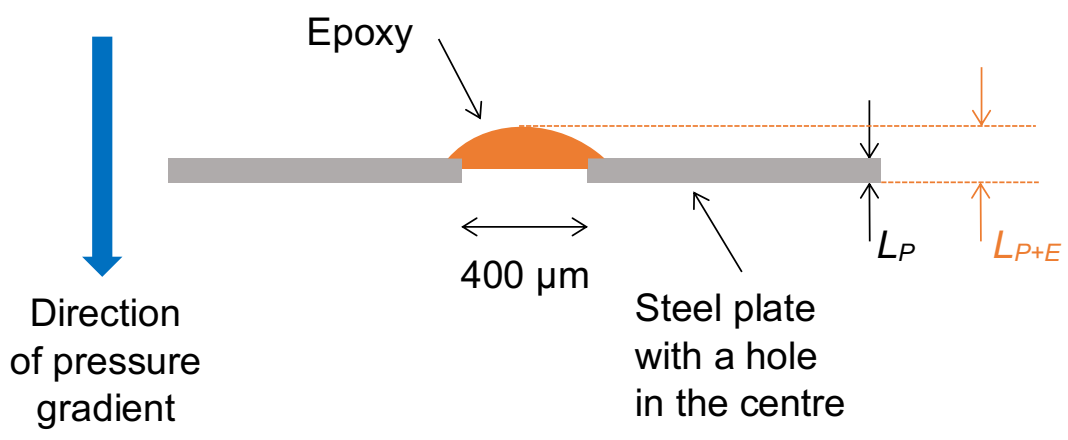

Fig. S8. A schematic side-view of the pure-epoxy membrane. $L_{p}$ and $L_{P+E}$ denote the thickness of steel plate and the combined thickness and steel plate and epoxy respectively. We approximated the thickness of the epoxy membrane by $\left(L_{P+E}-L_{P}\right)$, neglecting the thickness of epoxy that seeped through the hole. 


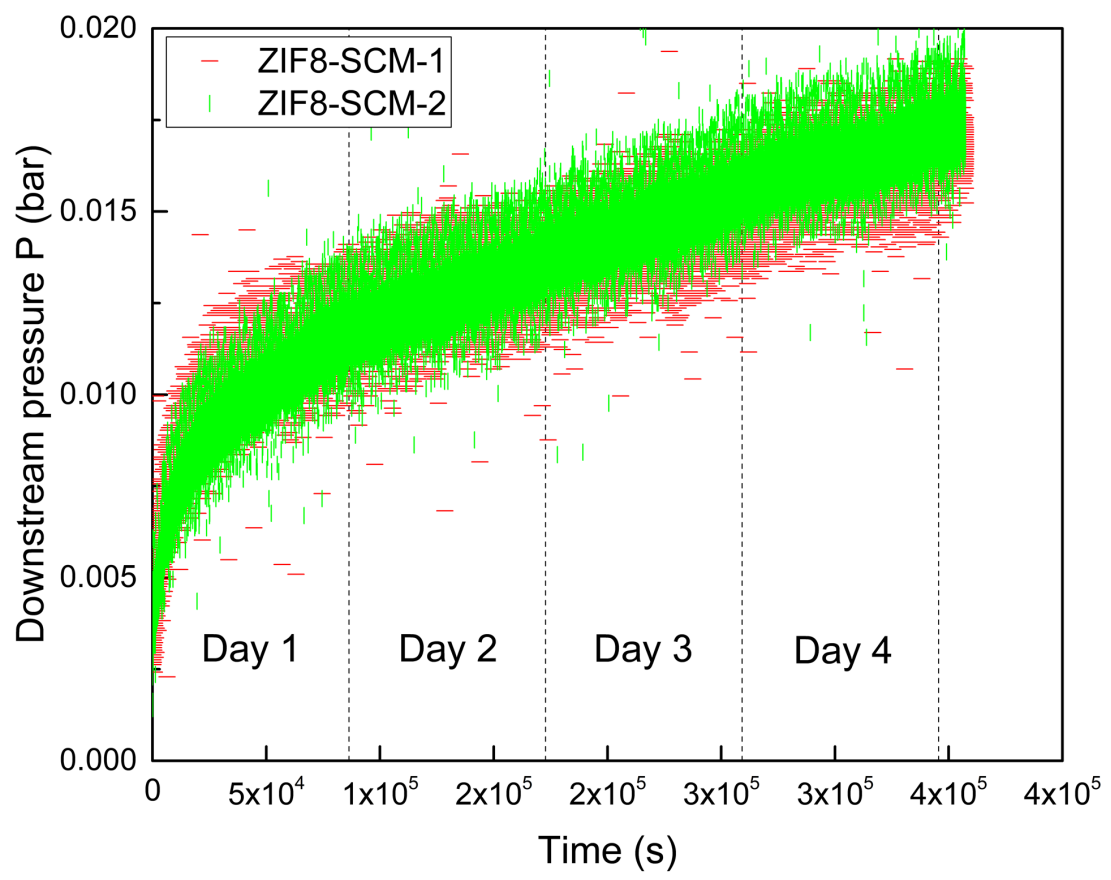

Fig. S9. Downstream pressure evolution due to air ingress. The air ingress for both ZIF8SCM-1 and ZIF8-SCM-2 reached steady-state after approximately 1.5 days (indicated by the linear portions of the two curves). We regarded the gradients of the linear portions as the ingress rate $(d P / d t)_{\text {ingress }}$ of the two membranes, and subtracted $(d P / d t)_{\text {ingress }}$ from the observed gradients during gas permeations $(d P / d t)_{\text {observed }}$ to obtain the rate of pressure rise actually caused by gas permeation. 


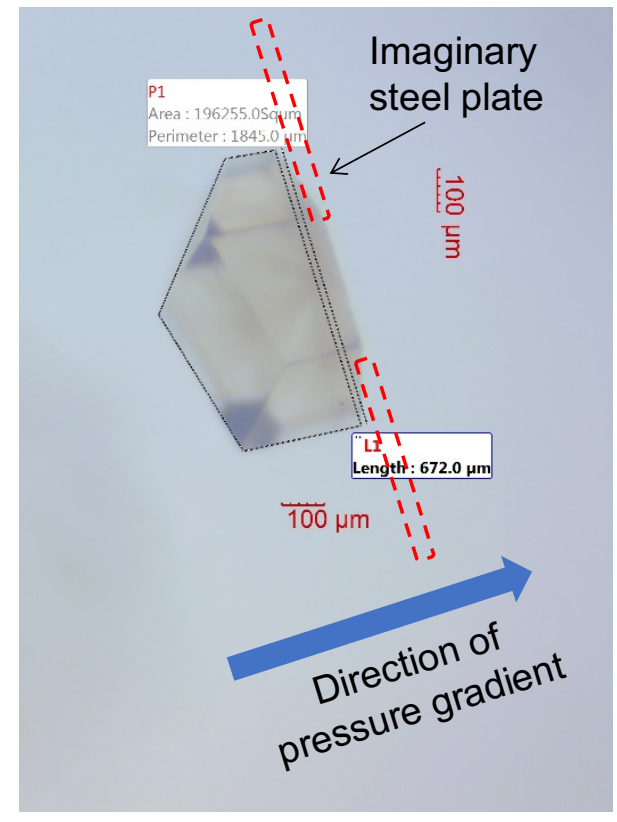

Fig. S10. Method of estimating the average crystal thickness. The crystal is the same as Fig. $3 b$ inset. 


\section{Supplementary tables}

Table S1. Single-gas permeabilities and ideal selectivities. Single-gas permeabilities $P$ of two single-crystal ZIF-8 membranes tested at room temperature $\left(20-25^{\circ} \mathrm{C}\right)$, and ideal permselectivities $\alpha(i / j)$ calculated as $P_{i} / P_{j}$. The transmembrane pressure difference was approximately constant at 2 bar.

\begin{tabular}{|l|r|r|}
\hline \multicolumn{3}{|l|}{ Single-gas permeability (barrer) } \\
\hline & ZIF8-SCM-1 & ZIF8-SCM-2 \\
\hline $\mathrm{He}$ & 1935 & 2309 \\
\hline $\mathrm{CO}_{2}$ & 750.7 & 577.1 \\
\hline $\mathrm{N}_{2}$ & 26.0 & 29.7 \\
\hline $\mathrm{CH}$ & 93.4 & 57.5 \\
\hline Ideal selectivity & \multicolumn{2}{|}{} \\
\hline$\alpha\left(\mathrm{He} / \mathrm{CO}_{2}\right)$ & 2.6 & 4.0 \\
\hline$\alpha\left(\mathrm{He} / \mathrm{N}_{2}\right)$ & 74.5 & 77.7 \\
\hline$\alpha\left(\mathrm{He} / \mathrm{CH}_{4}\right)$ & 20.7 & 40.1 \\
\hline$\alpha\left(\mathrm{CO}_{2} / \mathrm{N}_{2}\right)$ & 28.9 & 19.4 \\
\hline$\alpha\left(\mathrm{CO}_{2} / \mathrm{CH}_{4}\right)$ & 8.0 & 10.0 \\
\hline$\alpha\left(\mathrm{CH}_{4} / \mathrm{N}_{2}\right)$ & 3.6 & 1.9 \\
\hline
\end{tabular}


Table S2. Ceramic-supported polycrystalline ZIF-8 membranes reported in the literature and their single-gas permeances at room temperature. Permeabilities were calculated from permeances and membrane thicknesses.

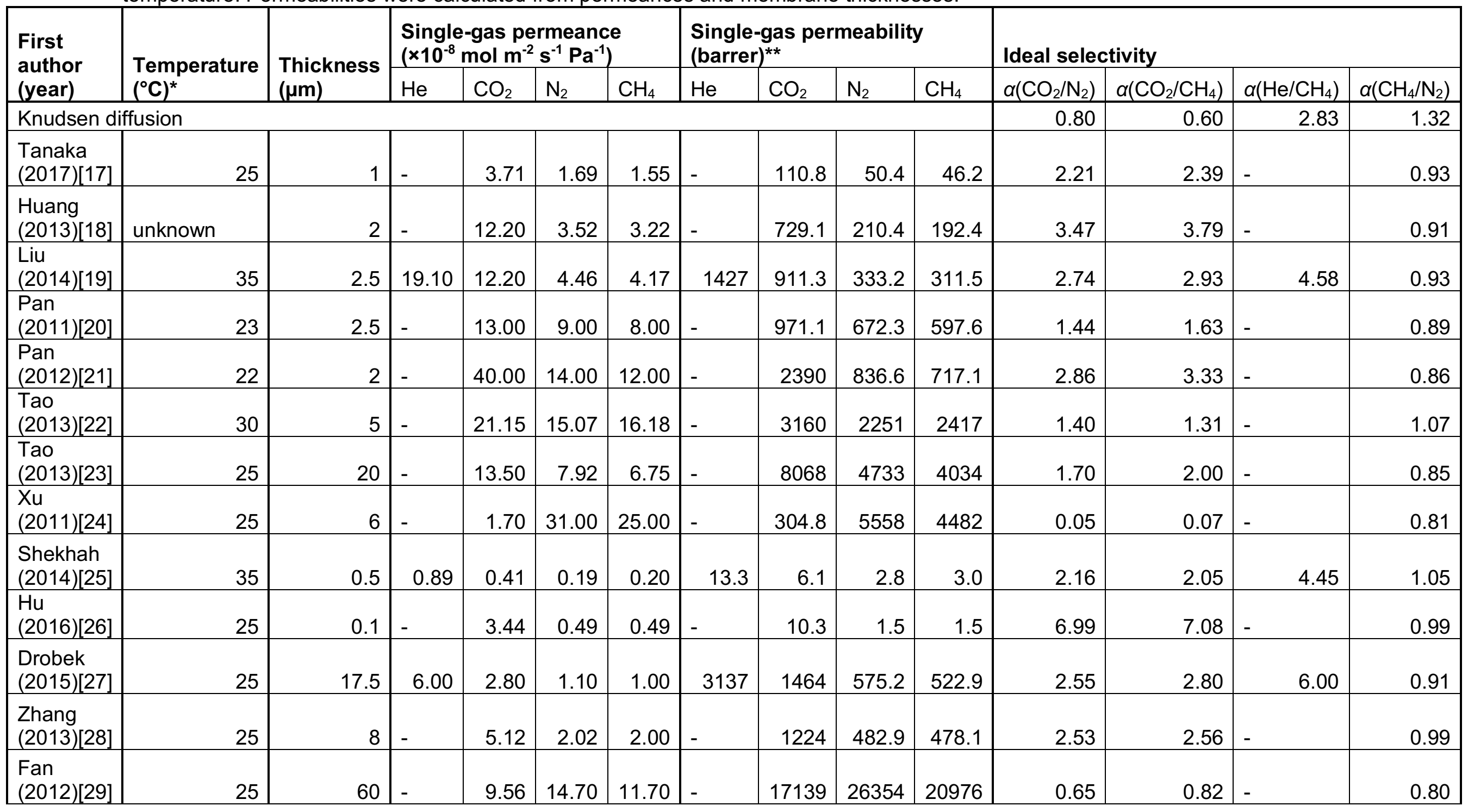




\begin{tabular}{|c|c|c|c|c|c|c|c|c|c|c|c|c|c|c|}
\hline \multirow{2}{*}{$\begin{array}{l}\text { First } \\
\text { author } \\
\text { (year) }\end{array}$} & \multirow{2}{*}{$\begin{array}{l}\text { Temperature } \\
\left({ }^{\circ} \mathrm{C}\right)\end{array}$} & \multirow{2}{*}{$\begin{array}{l}\text { Thickness } \\
(\mu \mathrm{m})\end{array}$} & \multicolumn{4}{|c|}{$\begin{array}{l}\text { Single-gas permeance } \\
\left(\times 10^{-8} \mathrm{~mol} \mathrm{~m}^{-2} \mathrm{~s}^{-1} \mathrm{~Pa}^{-1}\right)\end{array}$} & \multicolumn{4}{|c|}{$\begin{array}{l}\text { Single-gas permeability } \\
\text { (barrer) }\end{array}$} & \multicolumn{4}{|c|}{ Ideal selectivity } \\
\hline & & & $\mathrm{He}$ & $\mathrm{CO}_{2}$ & $\mathrm{~N}_{2}$ & $\mathrm{CH}_{4}$ & $\mathrm{He}$ & $\mathrm{CO}_{2}$ & $\mathrm{~N}_{2}$ & $\mathrm{CH}_{4}$ & $\alpha\left(\mathrm{CO}_{2} / \mathrm{N}_{2}\right)$ & $\alpha\left(\mathrm{CO}_{2} / \mathrm{CH}_{4}\right)$ & $\alpha\left(\mathrm{He} / \mathrm{CH}_{4}\right)$ & $\alpha\left(\mathrm{CH}_{4} / \mathrm{N}_{2}\right)$ \\
\hline $\begin{array}{l}\text { Yu } \\
(2016)[30]\end{array}$ & $e_{1}$ & 2.5 & - & 12.00 & 7.00 & 6.10 & - & 896.4 & 522.9 & 455.7 & 1.71 & 1.97 & $c_{1}$ & 0.87 \\
\hline $\begin{array}{l}\text { Hara } \\
(2015)[31]\end{array}$ & 25 & 20 & 19.00 & 10.00 & 4.50 & 5.00 & 11354 & 5976 & 2689 & 2988 & 2.22 & 2.00 & 3.80 & 1.11 \\
\hline $\begin{array}{l}\text { Hara } \\
(2014)[32]\end{array}$ & 25 & 80 & 4.50 & 2.70 & 0.95 & 1.10 & 10757 & 6454 & 2271 & 2629 & 2.84 & 2.45 & 4.09 & 1.16 \\
\hline $\begin{array}{l}\text { Wang } \\
(2016)[33]\end{array}$ & unspecified & 5 & - & 31.75 & 17.40 & 15.47 & - & 4744 & 2600 & 2311 & 1.82 & 2.05 & - & 0.89 \\
\hline $\begin{array}{l}\text { McCarthy } \\
(2010)[34]\end{array}$ & 25 & 20 & - & 4.45 & 1.49 & 1.33 & - & 2659 & 890.4 & 794.8 & 2.99 & 3.35 & - & 0.89 \\
\hline $\begin{array}{l}\text { Zhang } \\
(2017)[35]\end{array}$ & 25 & 0.55 & - & 1.47 & 3.16 & 1.66 & - & 24.1 & 51.8 & 27.3 & 0.47 & 0.88 & - & 0.53 \\
\hline $\begin{array}{l}\text { Liu } \\
(2015)[36]\end{array}$ & 30 & 0.16 & - & 0.19 & 0.06 & 0.02 & - & 0.9 & 0.3 & 0.1 & 3.09 & 10.29 & - & 0.30 \\
\hline $\begin{array}{l}\text { Bux } \\
(2009)[37]\end{array}$ & 25 & 30 & - & 1.33 & 0.52 & 0.48 & - & 1192 & 466.1 & 430.3 & 2.56 & 2.77 & - & 0.92 \\
\hline $\begin{array}{l}\text { Kong } \\
(2014)[38]\end{array}$ & 30 & 2.5 & - & 38.50 & 14.70 & 12.10 & - & 2876 & 1098 & 903.9 & 2.62 & 3.18 & - & 0.82 \\
\hline $\begin{array}{l}\text { Xie } \\
(2012)[39]\end{array}$ & 25 & 2 & - & 327.4 & 371.1 & - & - & 19567 & 22178 & - & 0.88 & - & - & - \\
\hline $\begin{array}{l}\text { Shah } \\
(2013)[40]\end{array}$ & 25 & 25 & - & 4.50 & 2.00 & 1.90 & - & 3362 & 1494 & 1419 & 2.25 & 2.37 & - & 0.95 \\
\hline
\end{tabular}

*: unless specified by authors, 'room temperature' is assumed to be $25^{\circ} \mathrm{C}$

**: 1 barrer $=10^{-10} \mathrm{~cm}^{3}(\mathrm{STP}) \mathrm{cm} \mathrm{cm}^{-2} \mathrm{~s}^{-1} \mathrm{~cm} \mathrm{Hg}^{-1}$. Permeability is calculated by the following equation:

Permeability $($ barrer $)=$ permeance $\left(\mathrm{mol} \mathrm{m}^{-2} \mathrm{~s}^{-1} \mathrm{~Pa}^{-1}\right) \times$ thickness $(\mu \mathrm{m}) \times 29.88$ 
Table S3. Crystallographic data of ZIF-8 from single-crystal X-ray diffraction.

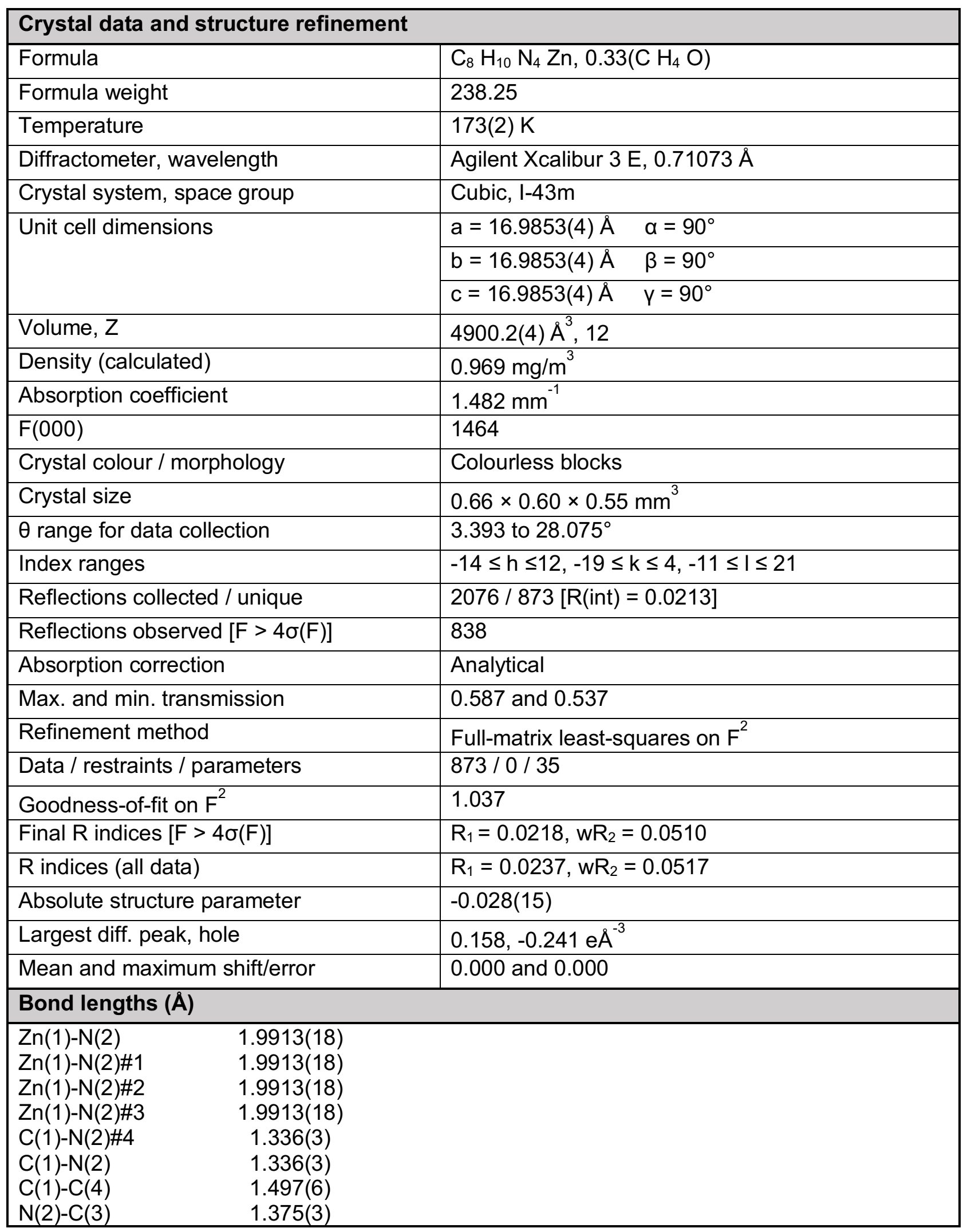




\begin{tabular}{|lc|}
\hline $\mathrm{C}(3)-\mathrm{C}(3) \# 4$ & $1.332(5)$ \\
\hline Bond angles ( ${ }^{\circ}$ & \\
\hline $\mathrm{N}(2)-\mathrm{Zn}(1)-\mathrm{N}(2) \# 1$ & $109.47(6)$ \\
$\mathrm{N}(2)-\mathrm{Zn}(1)-\mathrm{N}(2) \# 2$ & $109.48(6)$ \\
$\mathrm{N}(2) \# 1-\mathrm{Zn}(1)-\mathrm{N}(2) \# 2$ & $109.46(12)$ \\
$\mathrm{N}(2)-\mathrm{Zn}(1)-\mathrm{N}(2) \# 3$ & $109.46(12)$ \\
$\mathrm{N}(2) \# 1-\mathrm{Zn}(1)-\mathrm{N}(2) \# 3$ & $109.48(6)$ \\
$\mathrm{N}(2) \# 2-\mathrm{Zn}(1)-\mathrm{N}(2) \# 3$ & $109.48(6)$ \\
$\mathrm{N}(2) \# 4-\mathrm{C}(1)-\mathrm{N}(2)$ & $112.0(4)$ \\
$\mathrm{N}(2) \# 4-\mathrm{C}(1)-\mathrm{C}(4)$ & $123.98(19)$ \\
$\mathrm{N}(2)-\mathrm{C}(1)-\mathrm{C}(4)$ & $123.98(19)$ \\
$\mathrm{C}(1)-\mathrm{N}(2)-\mathrm{C}(3)$ & $105.3(3)$ \\
$\mathrm{C}(1)-\mathrm{N}(2)-\mathrm{Zn}(1)$ & $128.1(2)$ \\
$\mathrm{C}(3)-\mathrm{N}(2)-\mathrm{Zn}(1)$ & $126.63(16)$ \\
$\mathrm{C}(3) \# 4-\mathrm{C}(3)-\mathrm{N}(2)$ & $108.73(14)$ \\
\hline
\end{tabular}


Table S4. Lennard-Jones parameters for $\mathrm{CH}_{4}, \mathrm{CO}_{2}$ and $\mathrm{N}_{2}$ molecules. ${ }^{*}$

\begin{tabular}{|l|l|l|l|l|}
\hline Molecule & Atom & $\boldsymbol{\varepsilon} / \mathbf{k}_{\mathbf{B}}(\mathbf{K})$ & $\boldsymbol{\sigma}(\boldsymbol{A})$ & Charge \\
\hline $\mathrm{CH}_{4}$ & $\mathrm{CH}_{4}$ & 148.0 & 3.73 & N/A \\
\hline \multirow{2}{*}{$\mathrm{CO}_{2}$} & $\mathrm{C}$ & 27.0 & 2.80 & 0.7 \\
\cline { 2 - 5 } & $\mathrm{O}$ & 79.0 & 3.05 & -0.35 \\
\hline \multirow{2}{*}{$\mathrm{N}_{2}$} & $\mathrm{~N}$ & 36.0 & 3.31 & -0.482 \\
\cline { 2 - 5 } & N_com & N/A & N/A & 0.964 \\
\hline
\end{tabular}

* The Lorentz-Berthelot mixing rules were used to calculate the interaction parameters between unlike atoms.

Table S5. Predicted number of $\mathrm{CH}_{4}, \mathrm{CO}_{2}$ and $\mathrm{N}_{2}$ molecules adsorbed per $2 \times 2 \times 2 \mathrm{ZIF}-8$ system at 2 bars and $298 \mathrm{~K}$ for different ZIF-8 force fields from GCMC simulations.

\begin{tabular}{|l|l|l|l|}
\hline Force field used & $\mathbf{C H}_{\mathbf{4}}$ & $\mathbf{C O}_{\mathbf{2}}$ & $\mathbf{N}_{\mathbf{2}}$ \\
\hline Krokidas et al. & 21.14 & 45.72 & 7.42 \\
\hline Wu et al. & 12.07 & 20.71 & 4.77 \\
\hline Zhang et al. & 9.04 & 15.27 & 3.88 \\
\hline Zheng et al. & 20.88 & 48.88 & 7.42 \\
\hline
\end{tabular}

Table S6. Self-diffusion coefficients of $\mathrm{CH}_{4}, \mathrm{CO}_{2}$ and $\mathrm{N}_{2}$ in ZIF-8 calculated for loadings obtained at 2 bars and $298 \mathrm{~K}$ from MD simulations using different ZIF-8 force fields.

\begin{tabular}{|l|l|l|l|}
\hline Force field used & $\mathrm{CH}_{\mathbf{4}}\left(\mathbf{1 0}^{-10} \mathrm{~m}^{\mathbf{2}} / \mathbf{s}\right)$ & $\mathbf{C O}_{\mathbf{2}}\left(\mathbf{1 0}^{-10} \mathrm{~m}^{2} / \mathbf{s}\right)$ & $\mathbf{N}_{\mathbf{2}}\left(\mathbf{1 0}^{-10} \mathbf{~ m}^{\mathbf{2}} / \mathbf{s}\right)$ \\
\hline Krokidas et al. & 2.00 & 11.7 & 8.38 \\
\hline Wu et al. & 23.7 & 20.9 & 37.5 \\
\hline Zhang et al. & 0.37 & 3.31 & 1.53 \\
\hline Zheng et al. & 0.15 & 2.17 & 1.71 \\
\hline
\end{tabular}


Table S7. Simulated ideal selectivities of $\mathrm{CO}_{2} / \mathrm{N}_{2}, \mathrm{CO}_{2} / \mathrm{CH}_{4}$ and $\mathrm{CH}_{4} / \mathrm{N}_{2}$ mixtures in $\mathrm{ZIF}-8$. Here the ideal selectivity is defined as $\left(q / q_{j}\right) \times\left(D_{i} / D_{j}\right)$, where $q$ is the adsorbed amount and $D$ is the self-diffusion coefficient.

\begin{tabular}{|l|l|l|l|}
\hline Ideal Selectivity & $\mathbf{C O}_{\mathbf{2}} / \mathbf{N}_{\mathbf{2}}$ & $\mathbf{C O}_{\mathbf{2}} / \mathbf{C H}_{\mathbf{4}}$ & $\mathbf{C H}_{\mathbf{4}} / \mathbf{N}_{\mathbf{2}}$ \\
\hline Krokidas et al. & 8.61 & 12.65 & 0.68 \\
\hline Wu et al. & 2.42 & 1.51 & 1.60 \\
\hline Zhang et al. & 8.52 & 15.12 & 0.56 \\
\hline Zheng et al. & 8.36 & 33.87 & 0.25 \\
\hline
\end{tabular}




\section{References}

[1] G. Ramu, M. Lee, H.K. Jeong, Effects of zinc salts on the microstructure and performance of zeolitic-imidazolate framework ZIF-8 membranes for propylene/propane separation,

Microporous Mesoporous Mater., 259 (2018) 155-162.

[2] A. Schejn, L. Balan, V. Falk, L. Aranda, G. Medjahdi, R. Schneider, Controlling ZIF-8 nanoand microcrystal formation and reactivity through zinc salt variations, CrystEngComm, 16 (2014) 4493-4500.

[3] J. Cravillon, C.A. Schroder, H. Bux, A. Rothkirch, J. Caro, M. Wiebcke, Formate modulated solvothermal synthesis of ZIF-8 investigated using time-resolved in situ X-ray diffraction and scanning electron microscopy, CrystEngComm, 14 (2012) 492-498.

[4] J. Cravillon, R. Nayuk, S. Springer, A. Feldhoff, K. Huber, M. Wiebcke, Controlling Zeolitic Imidazolate Framework Nano- and Microcrystal Formation: Insight into Crystal Growth by TimeResolved In Situ Static Light Scattering, Chem. Mater., 23 (2011) 2130-2141.

[5] Q. Song, S.K. Nataraj, M.V. Roussenova, J.C. Tan, D.J. Hughes, W. Li, P. Bourgoin, M.A. Alam, A.K. Cheetham, S.A. Al-Muhtaseb, E. Sivaniah, Zeolitic imidazolate framework (ZIF-8) based polymer nanocomposite membranes for gas separation, Energy Environ. Sci., 5 (2012) 8359-8369.

[6] B. Seoane, J. Coronas, I. Gascon, M.E. Benavides, O. Karvan, J. Caro, F. Kapteijn, J. Gascon, Metal-organic framework based mixed matrix membranes: a solution for highly efficient CO2 capture?, Chem. Soc. Rev., 44 (2015) 2421-2454.

[7] M.G. Martin, J.I. Siepmann, Transferable Potentials for Phase Equilibria. 1. United-Atom Description of n-Alkanes, J. Phys. Chem. B, 102 (1998) 2569-2577.

[8] P.J. J., S.J. Ilja, Vapor-liquid equilibria of mixtures containing alkanes, carbon dioxide, and nitrogen, AIChE J., 47 (2001) 1676-1682.

[9] P. Krokidas, M. Castier, S. Moncho, E. Brothers, I.G. Economou, Molecular Simulation Studies of the Diffusion of Methane, Ethane, Propane, and Propylene in ZIF-8, J. Phys. Chem. C, 119 (2015) 27028-27037.

[10] X. Wu, J. Huang, W. Cai, M. Jaroniec, Force field for ZIF-8 flexible frameworks: atomistic simulation of adsorption, diffusion of pure gases as $\mathrm{CH} 4, \mathrm{H} 2, \mathrm{CO} 2$ and N2, RSC Advances, 4 (2014) 16503-16511.

[11] L. Zhang, Z. Hu, J. Jiang, Sorption-Induced Structural Transition of Zeolitic Imidazolate Framework-8: A Hybrid Molecular Simulation Study, J. Am. Chem. Soc., 135 (2013) 3722-3728.

[12] B. Zheng, M. Sant, P. Demontis, G.B. Suffritti, Force Field for Molecular Dynamics Computations in Flexible ZIF-8 Framework, J. Phys. Chem. C, 116 (2012) 933-938.

[13] B. Zheng, M. Sant, P. Demontis, G.B. Suffritti, Correction to "Force Field for Molecular Dynamics Computations in Flexible ZIF-8 Framework", J. Phys. Chem. C, 117 (2013) 2466224662.

[14] C. Zhang, R.P. Lively, K. Zhang, J.R. Johnson, O. Karvan, W.J. Koros, Unexpected Molecular Sieving Properties of Zeolitic Imidazolate Framework-8, J. Phys. Chem. Lett., 3 (2012) 2130-2134.

[15] K.S. Park, Z. Ni, A.P. Côté, J.Y. Choi, R. Huang, F.J. Uribe-Romo, H.K. Chae, M. O’Keeffe, O.M. Yaghi, Exceptional chemical and thermal stability of zeolitic imidazolate frameworks, Proc. Natl. Acad. Sci, 103 (2006) 10186-10191.

[16] D. Fairen-Jimenez, S.A. Moggach, M.T. Wharmby, P.A. Wright, S. Parsons, T. Düren, Opening the Gate: Framework Flexibility in ZIF-8 Explored by Experiments and Simulations, J. Am. Chem. Soc., 133 (2011) 8900-8902.

[17] S. Tanaka, K. Okubo, K. Kida, M. Sugita, T. Takewaki, Grain size control of ZIF-8 membranes by seeding-free aqueous synthesis and their performances in propylene/propane separation, J. Membr. Sci., 544 (2017) 306-311. 
[18] K. Huang, Z. Dong, Q. Li, W. Jin, Growth of a ZIF-8 membrane on the inner-surface of a ceramic hollow fiber via cycling precursors, Chem. Commun., 49 (2013) 10326-10328.

[19] D. Liu, X. Ma, H. Xi, Y.S. Lin, Gas transport properties and propylene/propane separation characteristics of ZIF-8 membranes, J. Membr. Sci., 451 (2014) 85-93.

[20] Y. Pan, Z. Lai, Sharp separation of C2/C3 hydrocarbon mixtures by zeolitic imidazolate framework-8 (ZIF-8) membranes synthesized in aqueous solutions, Chem. Commun., 47 (2011) $10275-10277$.

[21] Y. Pan, B. Wang, Z. Lai, Synthesis of ceramic hollow fiber supported zeolitic imidazolate framework-8 (ZIF-8) membranes with high hydrogen permeability, J. Membr. Sci., 421-422 (2012) 292-298.

[22] K. Tao, C. Kong, L. Chen, High performance ZIF-8 molecular sieve membrane on hollow ceramic fiber via crystallizing-rubbing seed deposition, Chem. Eng. J., 220 (2013) 1-5.

[23] K. Tao, L. Cao, Y. Lin, C. Kong, L. Chen, A hollow ceramic fiber supported ZIF-8 membrane with enhanced gas separation performance prepared by hot dip-coating seeding, J. Mater. Chem. A, 1 (2013) 13046-13049.

[24] G. Xu, J. Yao, K. Wang, L. He, P.A. Webley, C.-s. Chen, H. Wang, Preparation of ZIF-8 membranes supported on ceramic hollow fibers from a concentrated synthesis gel, J. Membr. Sci., 385-386 (2011) 187-193.

[25] O. Shekhah, R. Swaidan, Y. Belmabkhout, M. du Plessis, T. Jacobs, L.J. Barbour, I. Pinnau, M. Eddaoudi, The liquid phase epitaxy approach for the successful construction of ultra-thin and defect-free ZIF-8 membranes: pure and mixed gas transport study, Chem. Commun., 50 (2014) 2089-2092.

[26] Y. Hu, J. Wei, Y. Liang, H. Zhang, X. Zhang, W. Shen, H. Wang, Zeolitic Imidazolate Framework/Graphene Oxide Hybrid Nanosheets as Seeds for the Growth of Ultrathin Molecular Sieving Membranes, Angew. Chem. Int. Ed., 55 (2016) 2048-2052.

[27] M. Drobek, M. Bechelany, C. Vallicari, A. Abou Chaaya, C. Charmette, C. SalvadorLevehang, P. Miele, A. Julbe, An innovative approach for the preparation of confined ZIF-8 membranes by conversion of ZnO ALD layers, J. Membr. Sci., 475 (2015) 39-46.

[28] X. Zhang, Y. Liu, L. Kong, H. Liu, J. Qiu, W. Han, L.-T. Weng, K.L. Yeung, W. Zhu, A simple and scalable method for preparing low-defect ZIF-8 tubular membranes, J. Mater. Chem. A, 1 (2013) 10635-10638.

[29] L. Fan, M. Xue, Z. Kang, H. Li, S. Qiu, Electrospinning technology applied in zeolitic imidazolate framework membrane synthesis, J. Mater. Chem., 22 (2012) 25272-25276. [30] J. Yu, Y. Pan, C. Wang, Z. Lai, ZIF-8 membranes with improved reproducibility fabricated from sputter-coated ZnO/alumina supports, Chem. Eng. Sci., 141 (2016) 119-124.

[31] N. Hara, M. Yoshimune, H. Negishi, K. Haraya, S. Hara, T. Yamaguchi, ZIF-8 membranes prepared at miscible and immiscible liquid-liquid interfaces, Microporous Mesoporous Mater., 206 (2015) 75-80.

[32] N. Hara, M. Yoshimune, H. Negishi, K. Haraya, S. Hara, T. Yamaguchi, Diffusive separation of propylene/propane with ZIF-8 membranes, J. Membr. Sci., 450 (2014) 215-223.

[33] X. Wang, M. Sun, B. Meng, X. Tan, J. Liu, S. Wang, S. Liu, Formation of continuous and highly permeable ZIF-8 membranes on porous alumina and zinc oxide hollow fibers, Chem. Commun., 52 (2016) 13448-13451.

[34] M.C. McCarthy, V. Varela-Guerrero, G.V. Barnett, H.-K. Jeong, Synthesis of Zeolitic Imidazolate Framework Films and Membranes with Controlled Microstructures, Langmuir, 26 (2010) 14636-14641.

[35] S. Zhang, Z. Wang, H. Ren, F. Zhang, J. Jin, Nanoporous film-mediated growth of ultrathin and continuous metal-organic framework membranes for high-performance hydrogen separation, J. Mater. Chem. A, 5 (2017) 1962-1966. 
[36] Y. Liu, Y. Peng, N. Wang, Y. Li, J.H. Pan, W. Yang, J. Caro, Significantly Enhanced Separation using ZIF-8 Membranes by Partial Conversion of Calcined Layered Double Hydroxide Precursors, ChemSusChem, 8 (2015) 3582-3586.

[37] H. Bux, F. Liang, Y. Li, J. Cravillon, M. Wiebcke, J. Caro, Zeolitic Imidazolate Framework Membrane with Molecular Sieving Properties by Microwave-Assisted Solvothermal Synthesis, J. Am. Chem. Soc., 131 (2009) 16000-16001.

[38] L. Kong, X. Zhang, Y. Liu, S. Li, H. Liu, J. Qiu, K.L. Yeung, In situ fabrication of highpermeance ZIF-8 tubular membranes in a continuous flow system, Mater. Chem. Phys., 148 (2014) 10-16.

[39] Z. Xie, J. Yang, J. Wang, J. Bai, H. Yin, B. Yuan, J. Lu, Y. Zhang, L. Zhou, C. Duan, Deposition of chemically modified [small alpha]-Al2O3 particles for high performance ZIF-8 membrane on a macroporous tube, Chem. Commun., 48 (2012) 5977-5979.

[40] M. Shah, H.T. Kwon, V. Tran, S. Sachdeva, H.-K. Jeong, One step in situ synthesis of supported zeolitic imidazolate framework ZIF-8 membranes: Role of sodium formate, Microporous Mesoporous Mater., 165 (2013) 63-69. 